\title{
ORIGINALBEITRAG \\ Immobilienpreise und Immobilienzyklen und die Rolle von Angebotsbeschränkungen*
}

\author{
Christian A. L. Hilber
}

Online publiziert: 25. Oktober 2019

(C) Der/die Autor(en) 2019

Zusammenfassung Dieser Artikel untersucht die Auswirkungen von Angebotsbeschränkungen auf die Wachstumsrate und Zyklizität von Immobilienpreisen. Einfache Überlegungen zum Zusammenspiel von Angebot und Nachfrage lassen vermuten, dass Immobilienpreise dort stärker auf lokale Nachfrageschocks reagieren, wo es stärkere Bodennutzungsregulierungen und geografische Barrieren zur Erschließung und Bebauung von Flächen gibt, d.h. wo das Wohnimmobilienangebot preisunelastischer ist. Diese Vermutung wird durch die empirische Evidenz gestützt. Lokale Bodennutzungsregulierungen sind ein besonders entscheidender Faktor, welcher nicht nur lokale Unterschiede im Preiswachstum, sondern auch in der Preiszyklizität erklärt. Allerdings können sich starke Immobilienpreisaufschwünge auch in Standorten etwickeln, in denen die Bautätigkeit langfristig kaum eingeschränkt ist. Ein typisches Merkmal solcher Zyklen ist, dass der Preisverfall aufgrund erheblicher Überkapazitäten tendenziell ausgeprägter ist, als der Anstieg während der vorherigen Boomphase, so dass Immobilienpreise nach dem Preisverfall häufig niedriger sind als vor Beginn des Booms. Die Kurzsichtigkeit von Erschließungsunternehmen und Kreditgebern in Verknüpfung mit kurzfristigen Angebotsrigiditäten oder irrationale Übertreibungen (Euphorie unter Investoren) sind zwei plausible Erklärungen für dieses Phänomen.

Schlüsselwörter Immobilienpreise - Immobilienzyklen · Baulandangebot · Wohnungsangebot · Angebotsbeschränkungen · Raumplanung ·

Bodennutzungsregulierungen · Geografische Beschränkungen · Topografie · Angebotspreiselastizität · Einkommenspreiselastizität

C. A. L. Hilber $(\triangle)$

Department of Geography and Environment, London School of Economics, Houghton Street, London, WC2A 2 AE, Großbritannien

E-Mail: c.hilber@1se.ac.uk 


\title{
The impact of local supply constraints on house prices and price dynamics
}

\begin{abstract}
This article explores the role of supply constraints for house price growth and price dynamics. Simple demand-supply considerations suggest that house prices should respond more strongly to local housing demand shocks in places, in which regulatory restrictions and physical barriers to development are more binding, that is, the long run housing supply curve is more price inelastic. Empirical evidence supports this proposition. Labor demand- and credit supply-shocks cause house prices to respond more strongly in locations with tight long run barriers to development. Regulatory constraints are particularly important for explaining not only differential price growth but also differential cyclicality across locations. One apparent puzzle is that house price booms and busts occasionally occur in cities that allegedly have few long run barriers to development. One typical characteristic of such cycles is that, driven by significant overbuilding, the bust tends to be more pronounced than the preceding boom, leaving house prices often lower after the bust than before the start of the boom. Myopic developers and lenders in conjunction with short run construction lags or irrational exuberance (euphoria of investors) are two plausible explanations.
\end{abstract}

Keywords House prices - Real estate prices - Real estate cycles $\cdot$ House price cycles · Housing supply · Land supply · Supply constraints · Land use regulation · Geographical constraints · Topography $\cdot$ Supply price elasticity $\cdot$ Income price elasticity

\section{Einleitung}

In diesem Artikel wird die Rolle von Angebotsbeschränkungen als Determinante von Preisen und deren Dynamik auf Immobilienmärkten untersucht. Einfache theoretische Überlegungen legen nahe, dass, solange die Immobiliennachfragekurve einen fallenden Verlauf aufweist ${ }^{1}$, Immobilienpreise dort stärker auf lokale Immobiliennachfrageschocks reagieren, wo regulatorische Beschränkungen und bauliche Entwicklungshemmnisse (langfristige Angebotsbeschränkungen) bindender sind, d.h. das Immobilienangebot preisunelastischer (starrer) ist.

Abb. 1 verdeutlicht diesen Gedanken in einem einfachen Angebots-NachfrageModell, in dem zwei Extremfälle betrachtet werden: Eine Stadt A mit nur geringen Einschränkungen der Bautätigkeit und eine Stadt B mit einem starren Angebot. Aus der Abbildung lassen sich drei empirisch überprüfbare Vorhersagen ableiten:

\footnotetext{
1 Wenn Standorte von Natur aus unterschiedlich sind und Haushalte diese Unterschiede verschieden bewerten und/oder Haushalte sich in den Umzugskosten unterscheiden, dann weist die Nachfragekurve für das Wohnen an einem bestimmten Standort eine negative Steigung auf (vgl. dazu eine frühe Diskussion des Arguments von Arnott und Stiglitz [1979] und eine eingehendere Behandlung des Themas von Hilber [2017]).
} 
Abb. 1 Rolle der langfristigen Angebotsbeschränkungen. a Stadt A: Elastisches Angebot; b Stadt B: Nahezu perfekt unelastisches Angebot
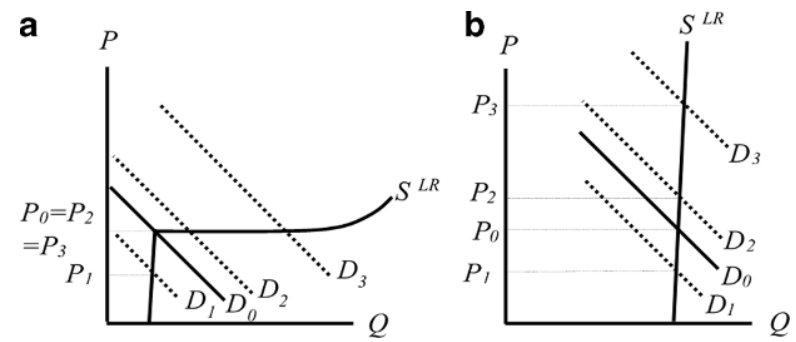

Hypothese 1 Immobilienpreise sollten angesichts positiver Nachfrageschocks (z.B. als Folge von positiven Arbeitsnachfrage- oder Hypothekarkreditangebotsschocks) in Städten mit starrem Angebot kurzfristig stärker steigen. Auch langfristig sollten Immobilienpreise in Städten mit begrenztem Angebot stärker steigen.

Hypothese 2 Durch Konjunkturzyklen (mit wirtschaftlichem Aufschwung und Abschwung) ausgelöste Immobilienpreiszyklen sollten in Städten mit langfristigen Angebotsbeschränkungen stärker ausgeprägt sein.

Hypothese 3 Bei stark negativen Nachfrageschocks sind sowohl kurz- als auch längerfristig kaum Mengen-, sondern ausschließlich (negative) Preisreaktionen zu erwarten. Angebotsbeschränkungen spielen keine Rolle.

Die letzte Hypothese ergibt sich, weil die Immobilienangebotskurve „,nach unten unelastisch“ ist (Glaeser und Gyourko 2005): Wohnraum ist ein äußerst langlebiges Gut und der Abriss von Gebäuden ist sehr kostspielig. Daher ist die Angebotskurve nahezu vollständig ${ }^{2}$ preisunelastisch wenn die Nachfrage nach Wohnimmobilien sinkt.

Die Rolle langfristiger Angebotsbeschränkungen als Determinanten der langfristigen Wohnimmobilienpreisentwicklung zu verstehen, ist von zentraler Bedeutung, sowohl für politische Entscheidungsträger als auch für Investoren.

Betrachten wir zunächst die politischen Entscheidungsträger. Verschiedenste nationale Politikmaßnahmen, Transfers oder Investitionen mit räumlich variablen Auswirkungen, lokale öffentliche Dienstleistungen, lokale Steuern, Steuertransfers über örtliche Gerichtsbarkeiten hinweg oder lokale Investitionen in die öffentliche Infrastruktur kapitalisieren sich allesamt mehr oder weniger in lokalen Immobilienpreisen. Inwieweit eine solche Kapitalisierung stattfindet und welche Verteilungseffekte sich daraus ergeben, hängt dabei vom Ausmaß lokaler Angebotsbeschränkungen ab. So wird zum Beispiel die staatliche Förderung einer armen Gemeinde bei einem starren Wohnungsangebot vollständig auf Mieten und Preise durchschlagen (Stadt B in Abb. 1). Sofern arme Haushalte zur Miete wohnen (und es keine strikte Mietpreiskontrolle gibt), könnte die Förderpolitik für sie ineffektiv sein: Die Regionalförderung führt zwar zur Verbesserung lokaler öffentlicher Dienstleistungen. Aus Sicht der örtlichen Mieter werden die Nutzen aber durch höhere Mieten vollständig kompensiert. Staatliche Förderung mit lokaler Zielausrichtung hilft den Armen

\footnotetext{
2 „Nahezu vollständig“, da der Wohnbestand an Wert verliert und irgendwann veraltet ist.
} 
also nicht unbedingt. Die wirklich Begünstigten könnten bereits wohlhabende und womöglich nicht ortsansässige Vermieter sein, sowie die typischerweise besser gestellten Wohneigentümer. Es könnte sogar einen Verdrängungseffekt geben, arme Mieter könnten gezwungen sein, an weniger beliebte Orte mit niedrigeren Mieten zu ziehen.

Das Verständnis der Dynamik der Immobilienpreise und ihrer Determinanten ist auch für Immobilieninvestoren wichtig, und insbesondere für potentielle Käufer von Eigenheimen. Tatsächlich ist die Entscheidung, wo und wann das erste Haus gekauft werden soll, womöglich die wichtigste finanzielle Entscheidung, welche Viele (und außerhalb Deutschlands und der Schweiz ${ }^{3}$ die meisten von uns) zu Lebzeiten treffen.

Stellen wir uns eine potenzielle Erstkäuferin vor, die erwägt, ihr gesamtes Vermögen von $50.000 €$ in eine Wohnung im Wert von $200.000 € \mathrm{zu}$ investieren. Die verbleibenden $150.000 €$ werden durch einen Immobilienkredit finanziert, der mit 3\% verzinst ist. Der Einfachheit halber abstrahieren wir von Tilgung, Inflation und Steuern. Wenn die Investorin Glück hat (oder diesen Artikel gelesen hat) und am Tiefpunkt des Zyklus kauft (d.h. zu Beginn einer Hochkonjunktur), könnte sich ihr Vermögen innerhalb weniger Jahre vervielfachen. Angenommen, der Preis für die Wohnung steigt über einen Zeitraum von fünf Jahren jährlich um $6 \%$ und die ,kalkulatorische Eigenmiete“ (Eigentümer können als Vermieter betrachtet werden, die an sich selbst vermieten) beträgt $4 \%$ des Werts der Wohnung (8000€ im ersten Jahr), dann liegt die Gesamtrendite der Wohnung bei $10 \%(=6 \%+4 \%)$. Innerhalb des Fünfjahreszeitraums wird die Wohnung einen Wert von $268.000 €$ $\left(=200.000 € \times 1,06^{5}\right)$ erreichen, was einem Kapitalgewinn von $68.000 €$ bei einer Erstinvestition von $50.000 €$ entspricht. Unter Berücksichtigung der Finanzierungskosten von $3 \%$ auf die $150.000 €$ Fremdkapital (75\% des Hauspreises) ergibt sich eine jährliche Eigenkapitalrendite von $31 \%$ [= $(10 \%-0,75 \times 3 \%): 0,25]$. Betrachten wir nun dieselbe Investition mit denselben Annahmen, mit der Ausnahme, dass die Immobilienpreise gerade ihren Höhepunkt erreicht haben und über einen Zeitraum von fünf Jahren jährlich um $6 \%$ fallen. Die Investorin erhält weiterhin die 4-prozentige Konsumrendite aus der kalkulatorischen Eigenmiete, sodass die Gesamtrendite der Immobilie $-2 \%$ beträgt. Diese Annahmen implizieren einen Kapitalverlust von rund $53.000 €\left[=200.000 € \times(1-0,06)^{5}-200.000 €\right]$ oder eine jährliche Eigenkapitalrendite von $-17 \%[=(-2 \%-0,75 \times 3 \%): 0,25]$. Dieser Verlust von mehr als $50.000 €$ bedeutet, dass die Anlegerin ihr gesamtes Vermögen verliert. Spätestens am Ende der Zinsbindung dürfte die Bank aufgrund des Wertverlustes nach weiteren Sicherheiten fragen. Gelingt es der Schuldnerin nicht, über die Laufzeit des Kredits signifikante Ersparnisse zu bilden, wird sie diese Sicherheiten nicht erbringen können und wird das Haus verlustreich verkaufen müssen. Das Verständnis der Funktionsweise der Immobilienmärkte - und insbesondere der Dynamik der

\footnotetext{
3 Deutschland und die Schweiz haben die mit Abstand niedrigsten Wohneigentumsquoten in Europa. Die Wohneigentumsquote in Deutschland liegt knapp über 50\%, in der Schweiz ist sie mit um die $40 \%$ sogar deutlich niedriger.
} 
Immobilienpreise - ist also sowohl für politische Entscheidungsträger als auch für Investoren von ganz entscheidender Bedeutung. ${ }^{4}$

Allerdings gibt es eine Reihe von Anmerkungen und Relativierungen. Erstens stellt Abb. 1 ein partielles Gleichgewicht dar, d.h. es bestehen keine Interaktionen mit anderen Güter- und Faktormärkten (z. B. dem Arbeitsmarkt). Es ist also nicht ganz klar, ob der Link zwischen Angebotsbeschränkungen und der Preiszyklizität auch in der Praxis so ausgeprägt vorhanden ist, wie von der Abbildung suggeriert, oder allenfalls von Rückkoppelungseffekten in anderen Märkten abgeschwächt wird.

Zweitens stellt sich die Frage, was die Ursachen unelastischer Angebotskurven sind. Wie wichtig sind insbesondere Bodennutzungsregulierung und geografisch bedingte Hindernisse für die Bautätigkeit? Ich befasse mich mit diesen Fragen in Abschn. 3, in dem ich Erkenntnisse aus der aktuellen empirischen Literatur zusammenfasse.

Drittens reagiert das Wohnungsangebot in der kurzen und langen Frist unterschiedlich. Kurzfristig ist das Wohnungsangebot auf Grund typischerweise mehrjähriger Planungs- und Bauphasen preisunelastisch. Während in manchen Städten das Wohnungsangebot auch langfristig (aufgrund regulatorischer und/oder geografischer Beschränkungen) unelastisch auf Preisänderungen reagiert, reagiert es in anderen Städten elastisch. Diese Unterschiede zwischen kurzfristiger und langfristiger Preiselastizität des Angebots wirken sich auf die Dynamik der Immobilienpreise aus. Ich werde dies in Abschn. 4 untersuchen und im letzten Abschnitt meine Schlüsse ziehen.

\section{Der zyklische Charakter von Wohnungsmärkten: Visualisierung}

Eines der auffälligsten Merkmale von lokalen Wohnungsmärkten - und Immobilienmärkten im Allgemeinen - ist, dass die Preise in der Regel zyklischer Natur sind: Aufschwung und Abschwung wiederholen sich in einem mehr oder weniger regelmäßigen Muster. Technisch ausgedrückt: Preisänderungen sind durch eine kurzfristige serielle Korrelation (serial correlation) und eine langfristige Rückkehr zum Mittelwert (mean reversion) gekennzeichnet, wodurch Immobilienpreise zumindest theoretisch vorhersehbar werden. ${ }^{5}$

Abb. 1 legt nahe, dass bei ähnlichen Nachfrageschocks die Preisausschläge in den Zyklen abhängig von der langfristigen Preiselastizität des Angebots erheblich vari-

\footnotetext{
${ }^{4}$ Eine wichtige Einschränkung ist hier die Tatsache, dass bei fallenden Immobilienpreisen nicht nur ,starter homes“ (Startwohnungen oder -häuser), sondern auch „trade-up homes“ (grossflächigere und qualitativ bessere Wohnungen und Häuser) erschwinglicher werden. Wohneigentum dient also der Absicherung von Miet- und Umzugsrisiken (Sinai und Souleles 2005, 2013). In unserem stilisierten Beispiel wird dies unserer Investorin natürlich wenig helfen, da ihr gesamtes Vermögen vernichtet wurde und ihr nun die notwendigen Mittel für ein „trade-up home“ fehlen. Die Absicherung hilft nur Immobilieneigentümern mit etwas Vermögen, die sich nicht stark kreditgestützt finanzieren und ihren Wohnraum durch den Kauf einer größeren Immobilie steigern möchten. Diese Haushalte profitieren möglicherweise sogar von fallenden Immobilienpreisen, da das größere „,trade-up home“ in absoluten Zahlen mehr an Wert verliert als das "starter home“.

${ }^{5}$ Für eine vertiefte Analyse der Merkmale der Hauspreisdynamik siehe z. B. Glaeser und Nathanson (2017).
} 


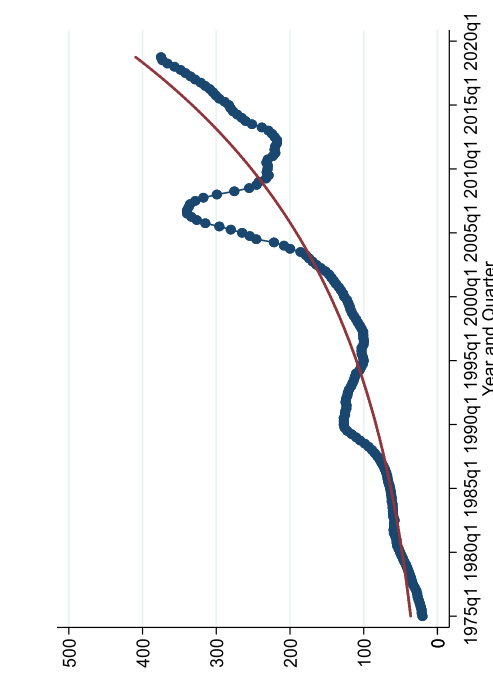

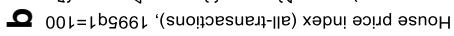
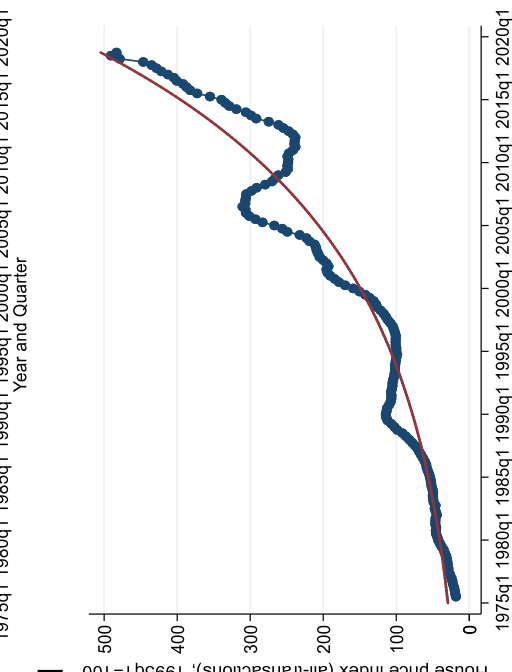

突至解

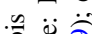

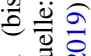

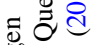

焉范

:

항 $\frac{0}{2}$

要专

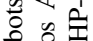

要,

安 2.

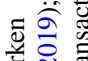

新

嵌 $\bar{\sigma}$

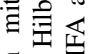

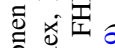

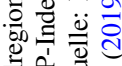

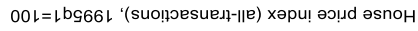

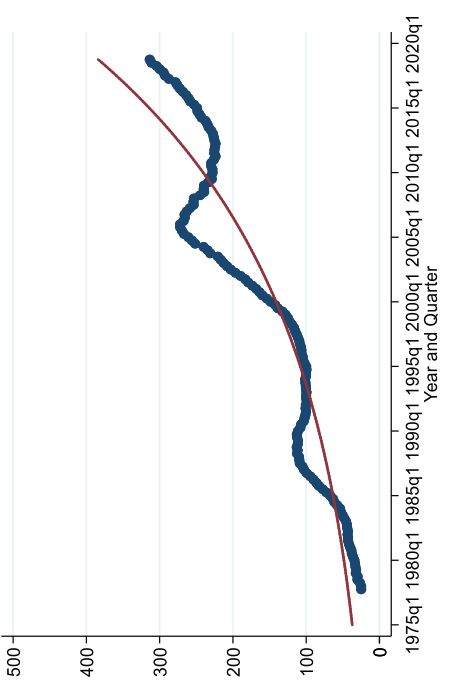

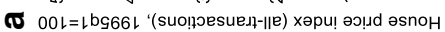

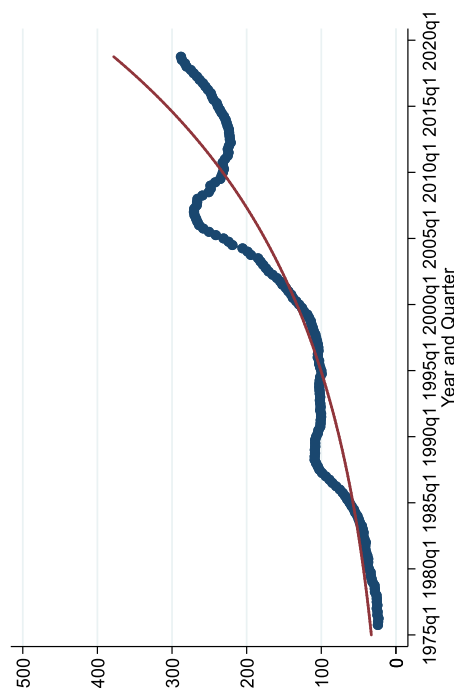

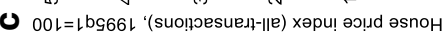
言主范

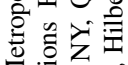

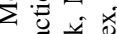

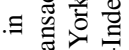
害站主 类乙的

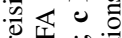
異前 可不

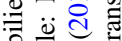
링 है 严过至 它希壱

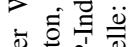
䐱总高

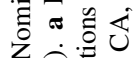
$\checkmark$ 焉总

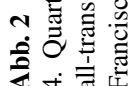




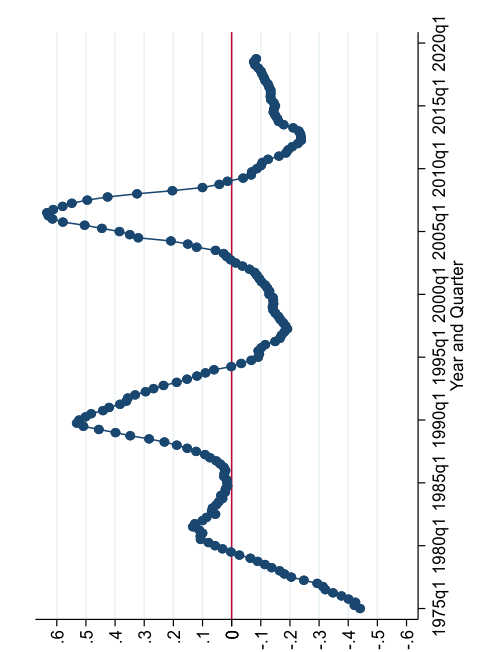

?

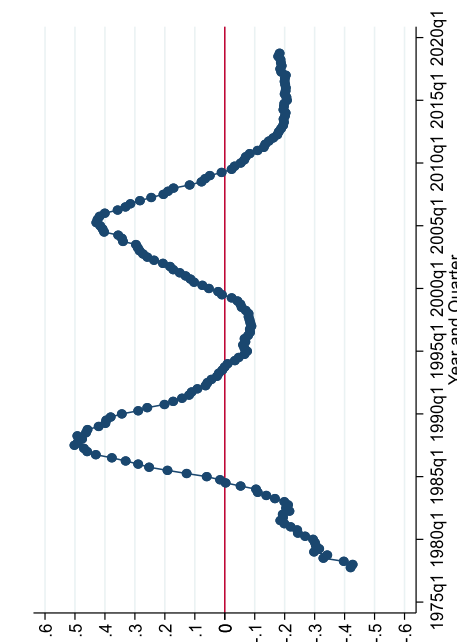

(ง

(

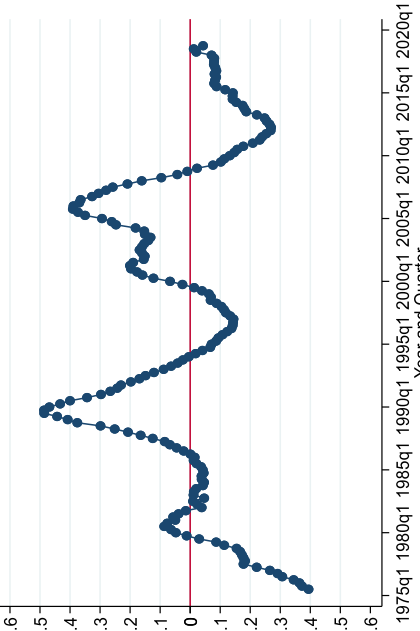

$\nabla$

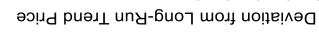

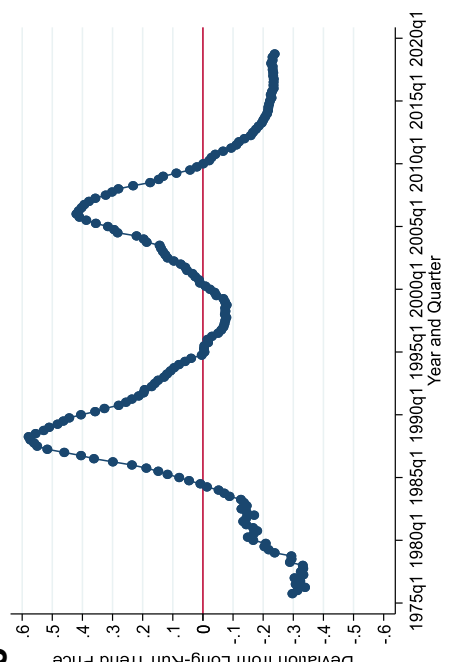

気这官

娄政

高主希方

ํㅗㅇ

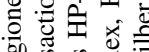

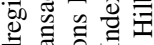

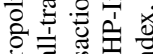

㐘的会

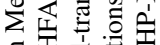

. 耎言总

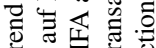

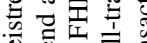

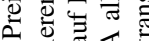

ข

苛吉吉

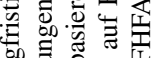

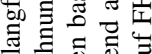

छ

$>$ Ф

.

D.

羊江品

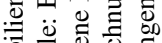

응.

吕绖

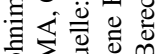

¿ $2 \infty_{00}$

3 ธิن 四

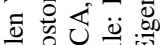

สี

응

के

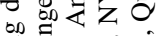

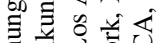

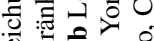

궁

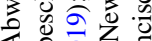

《气

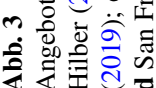




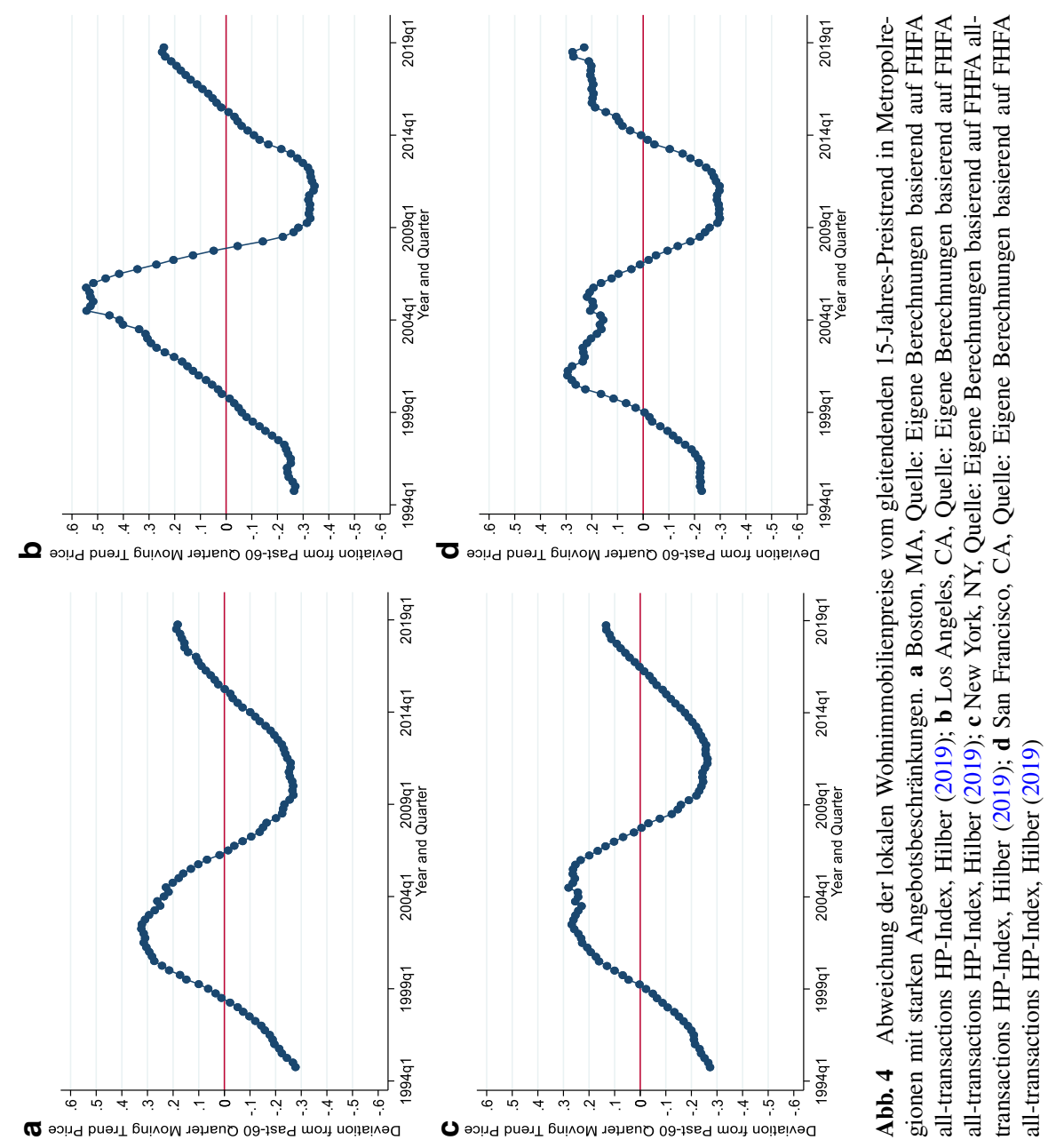


ieren sollten (Hypothese 2). Die Vereinigten Staaten von Amerika sind ein idealer Ort, um diese Prognose zu testen. Dies liegt daran, dass regulatorische und geografische Angebotsbeschränkungen und die entsprechende Preiselastizität des Angebots in den Metropolregionen sehr unterschiedlich sind.

Um den Zusammenhang empirisch aufzuzeigen, habe ich vier Metropolregionen ausgewählt, die bekanntermaßen strenge regulatorische und geografische Angebotsbeschränkungen und eine niedrige Preiselastizität des Angebots aufweisen: Boston (MA), Los Angeles (CA), New York (NY) und San Francisco (CA). ${ }^{6}$ Die Abb. 2, 3 und 4 veranschaulichen die Zyklizität dieser Immobilienmärkte anhand von drei alternativen Maßen. ${ }^{7}$ Abb. 2 zeigt die nominalen Immobilienpreisindizes (fette blaue Linien) sowie die semilogarithmischen ,gefitteten“ Regressionslinien (dünne dunkelrote Linien). Die Rohdaten liegen auch Abb. 3 und 4 zugrunde. In allen vier Märkten sind Phasen des Auf- und Abschwungs bereits bei der Betrachtung der Rohdaten klar erkennbar. Das zweite Maß ist die Abweichung der Immobilienpreise vom langfristigen Trend des lokalen Marktes. ${ }^{8}$ Der Trend wird dabei in einem semilogarithmischen Regressionsmodell über den gesamten Stichprobenzeitraum gebildet. Die vier Grafiken in Abb. 3 verdeutlichen, dass periodische WohnungspreisWachstumsraten im Zeitablauf korreliert sind ${ }^{9}$ aber tendenziell immer wieder zum Trendwachstum zurückkehren. Abb. 4 wiederum zeigt die Abweichung der Immobilienpreise vom gleitenden Trend der letzten 60 Quartale. Der gleitende Trend wird aus semilogarithmischen Regressionen gebildet, die für jedes Quartal separat durchgeführt werden. ${ }^{10}$ Diese Messung ähnelt der in Abb. 3 verwendeten, jedoch

\footnotetext{
${ }^{6}$ Hilber und Robert Nicoud (2013, S. 39) erstellen für eine Reihe bekannter Metropolregionen ein Ranking von Regulierungsbeschränkungen und der Knappheit von bebaubaren Grundstücken. San Francisco, Los Angeles, Boston und New York erzielen bei diesen Maßen alle eine hohe Punktzahl. Die verschiedenen im Ranking dokumentierten Maße leiten sich von Glaeser et al. (2005), Gyourko et al. (2008), Saks (2008), Saiz (2010) und Hilber und Robert-Nicoud (2013) ab.

7 Diese Abbildungen und die dazugehörigen Berechnungen stammen von Hilber (2019). Die Preisdaten stammen von der Federal Housing Finance Agency (FHFA).

8 Der Untersuchungszeitraum variiert zwischen den Metropolregionen etwas. Die Zeitreihe für Los Angeles begann im ersten Quartal 1975 (1975q1). Die Zeitreihen der anderen Märkte beginnen etwas später, in der Regel zwischen 1975q2 und 1980q1. Der Endzeitraum ist für alle Metropolregionen gleich: $2018 q 4$.

9 Perioden mit starkem Wachstum folgen auf Perioden mit starkem Wachstum (Überschwingen) und Perioden mit schwachem oder negativem Wachstum folgen auf Perioden mit schwachem oder negativem Wachstum (Unterschreitung).

10 Ein 60-Quartal- (oder 15-Jahres-) Fenster wurde ausgewählt, da in der Mehrheit der Metropolregionen in den Vereinigten Staaten mit klar definierten Zyklen um 1990 und erneut um 2005 Spitzenwerte und um 1995 und 2010 Tiefstwerte beobachtet wurden. Eine systematischere Analyse zeigt, dass klar definierte volle Zyklen in den 1980er und 1990er Jahren im Durchschnitt etwa 14 bis 15 Jahre dauerten, sich jedoch irgendwo zwischen 11 und 18 Jahren bewegten. Darüber hinaus gibt es sehr große Unterschiede in den Jahren des Aufschwungs und des Abschwungs in den einzelnen Metropolregionen, wobei einige Metropolregionen im selben Jahr, in dem andere Metropolregionen die Talsohle erreichen, einen Preisspitzenwert erreichen. Dies deutet darauf hin, dass es keinen ,nationalen Wohnungsmarkt“ gibt (zumindest nicht in größeren Ländern wie den Vereinigten Staaten). Die Wohnungsmärkte sind von lokaler Natur und werden von den lokalen Nachfrage- und Angebotsfaktoren bestimmt, wie ich im Folgenden genauer erläutern werde. Dies bedeutet jedoch nicht, dass aggregierte Preise auf nationaler Ebene keine zyklischen Merkmale aufweisen. Das liegt daran, dass Immobilienpreise auch durch makroökonomische Schocks getrieben werden und sich die lokalen Unterschiede gegenseitig ausgleichen können. Wenn die makroökonomischen Schocks sehr stark sind und das gesamte Land betreffen (z. B. die große Finanzkrise in den Vereinigten
} 


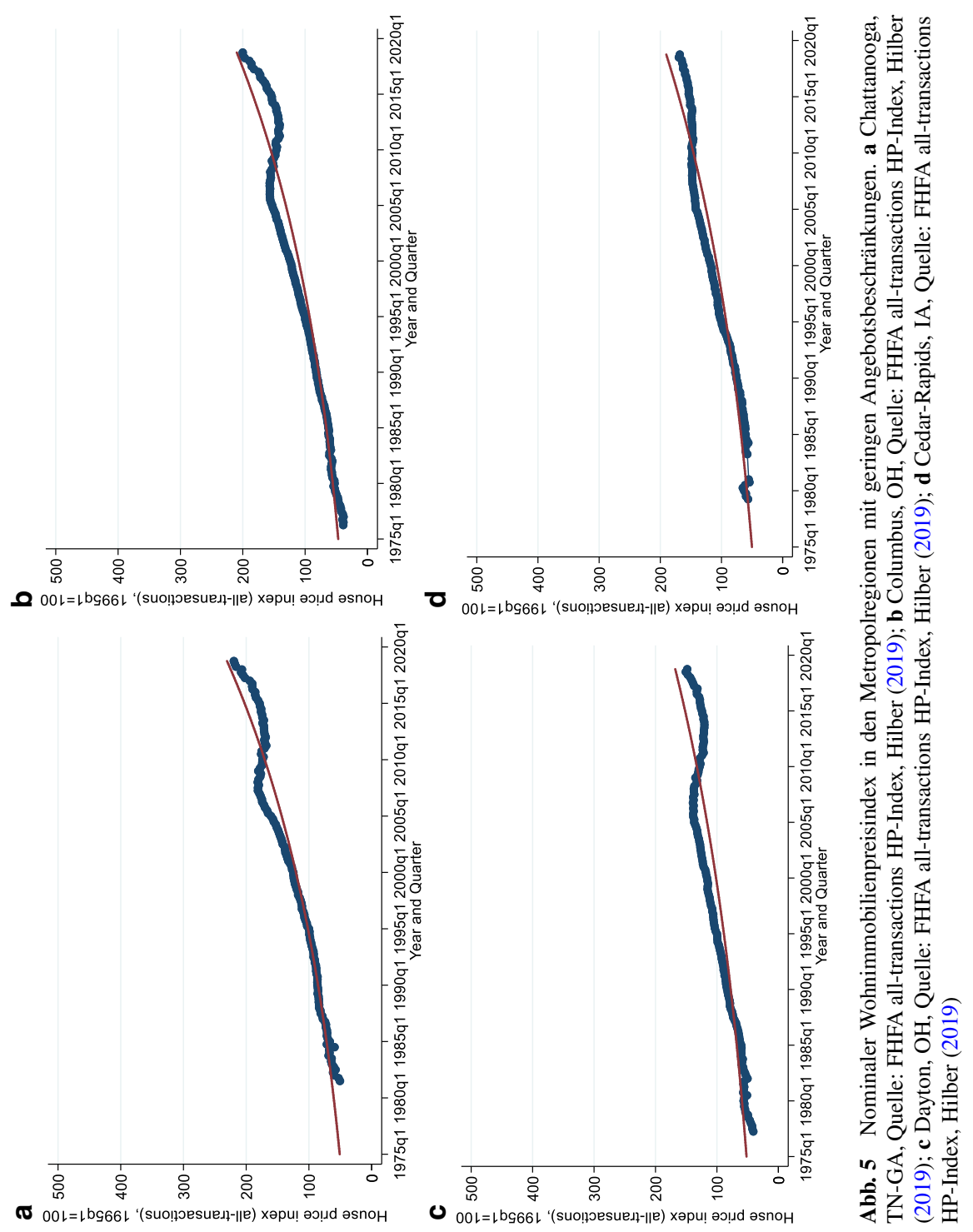




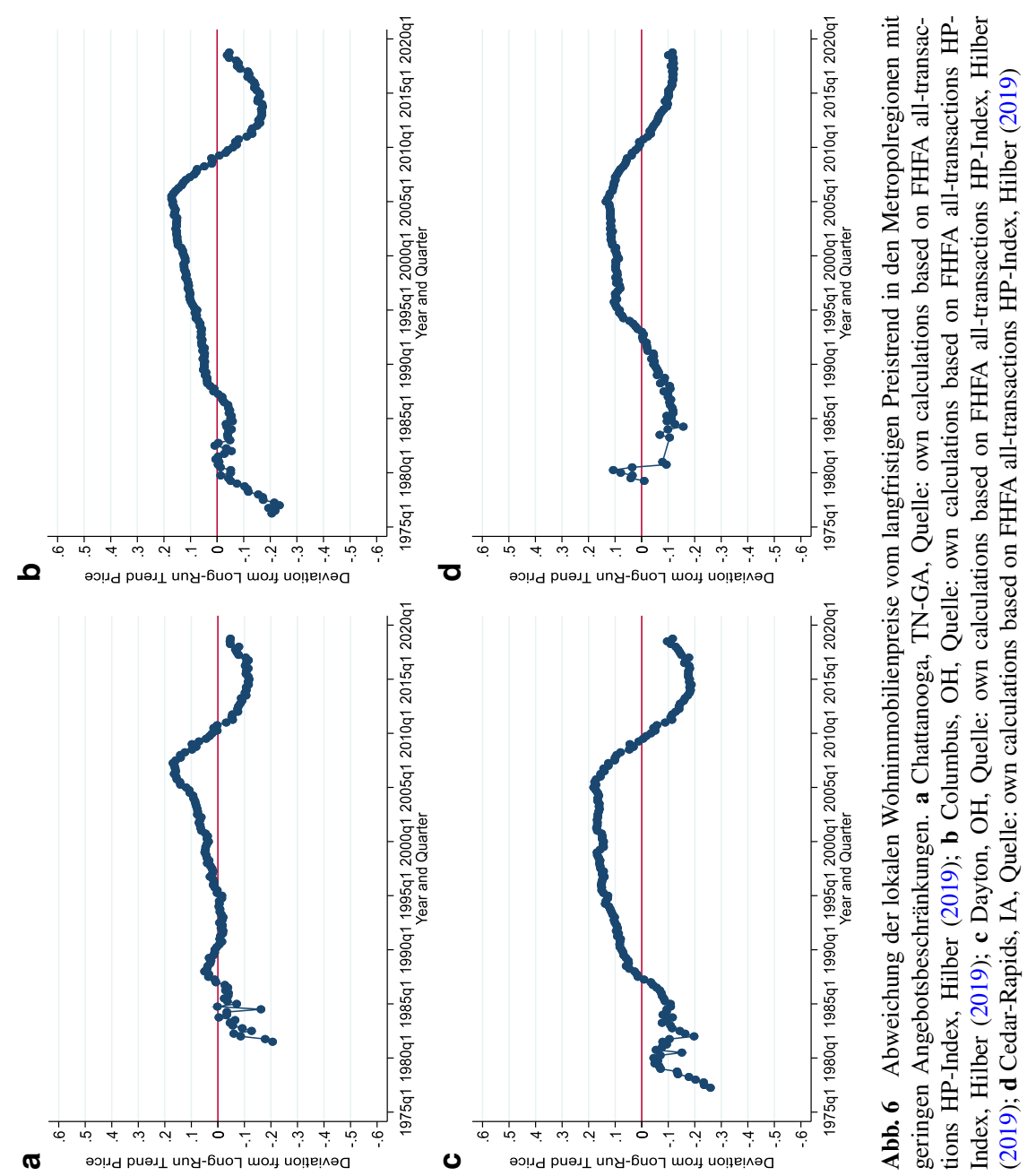




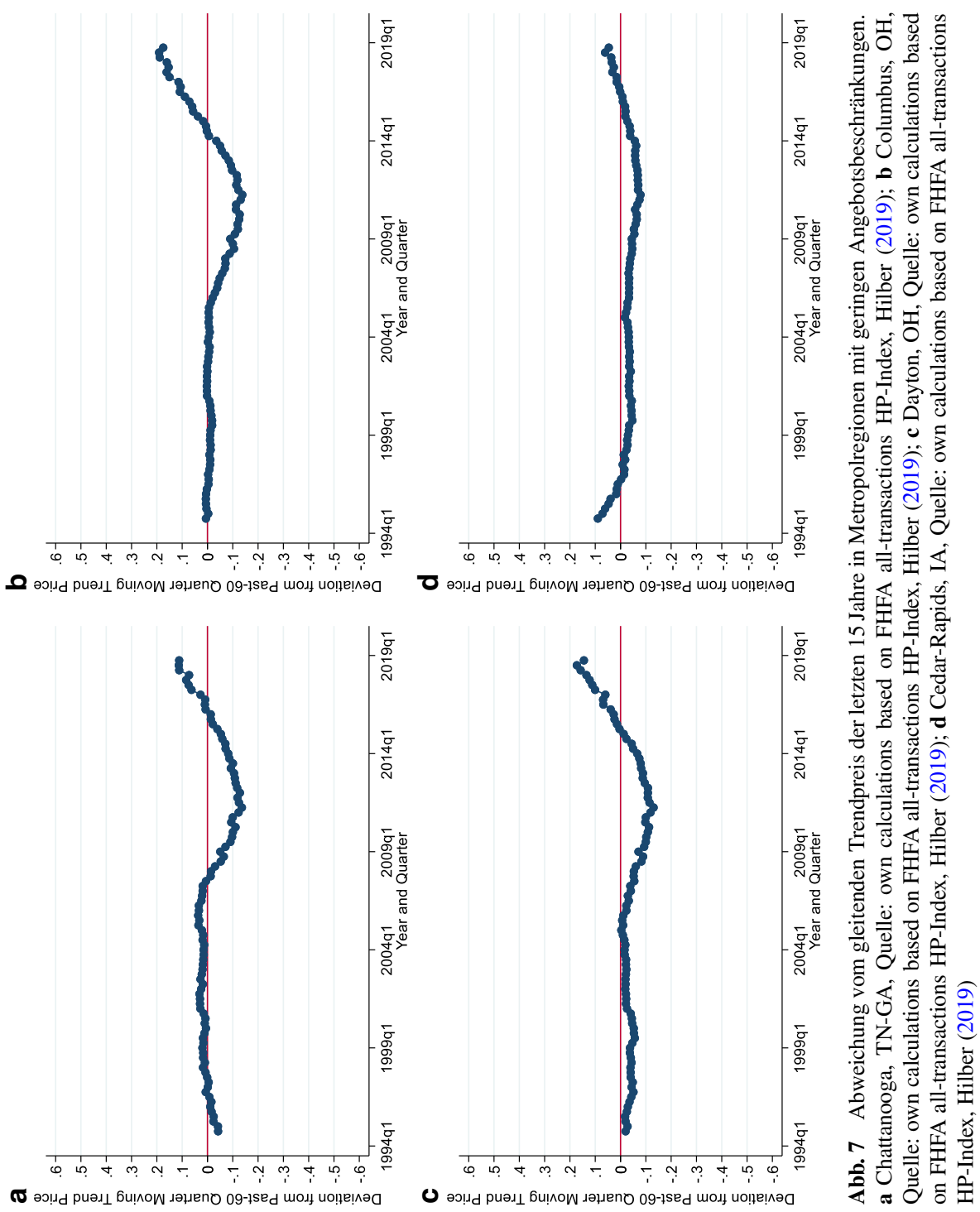


nutze ich in jedem Zeitraum nur zurückliegende Perioden (d.h. Informationen, die für Prognosezwecke verfügbar sind). Auch hier deuten alle vier Grafiken auf stark zyklische lokale Immobilienmärkte hin.

Betrachten wir als nächstes vier Metropolregionen, die über ausreichend bebaubares Land für Wohnzwecke und flexible Bestimmungen zur Landnutzung verfügen. ${ }^{11}$ Diese Metropolregionen sind: Chattanooga (TN), Columbus $(\mathrm{OH})$, Dayton $(\mathrm{OH})$ und Cedar-Rapids (IA). Die Abb. 5, 6 und 7 entsprechen den Abb. 2, 3 und 4 für die angebotsunelastischen Märkte. Abb. 5 zeigt, dass in keinem der vier Märkte eine Zyklizität erkennbar ist. Bis Mitte der 2000er Jahre ist das Wachstum der Immobilienpreise langsam, aber stetig. Die Preise fallen dann leicht oder bleiben während der großen Finanzkrise stabil und erholen sich Anfang der 2010er Jahre. Abb. 6 zeigt, dass die Immobilienpreise in den 1970er, 1980er und in einigen Fällen in den frühen 1990er Jahren leicht unterhalb des langfristigen Trends lagen. In den späten 1990er Jahren bis Mitte der 2000er Jahre lagen die Preise dagegen leicht über dem langfristigen Trend. In der Folge fielen sie während und nach der großen Finanzkrise wieder etwas unter den langfristigen Trend. Keiner der Märkte zeigt jedoch ein auffällig zyklisches Verhalten. Abb. 7 zeigt schließlich, dass die Immobilienpreise in allen vier Märkten bis etwa 2005/6 in etwa dem gleitenden Trend der letzten 15 Jahre folgten. Dies deutet darauf hin, dass die Immobilienpreise unzyklisch sind, was mit einer hochelastischen langfristigen Angebotskurve (und Hypothesen 1 und 2) vereinbar ist. Ab 2005/6 liegen die Preise jedoch unter dem gleitenden Trendpreis. Dies steht im Einklang mit einer Angebotskurve, die nach unten starr ist: Die Preise wachsen in Perioden mit stabiler oder wachsender Nachfrage mit einer Rate die dem Abzinsungssatz entspricht. In Perioden mit negativen Nachfrageverschiebungen (z.B. in den Jahren der großen Finanzkrise) werden die Preise jedoch zwangsläufig fallen, selbst in Märkten mit einem hochelastischen Angebot (Hypothese 3).

Die graphische Evidenz in den Abb. 2, 3, 4, 5, 6 und 7 stützt die These, dass die Dynamik der Immobilienpreise auf lokaler Ebene von den lokalen Nachfrage- und Angebotsfaktoren bestimmt wird und, dass die Zyklizität der Immobilienpreise vor allem von der langfristigen Preiselastizität des Angebots auf dem Markt bestimmt wird.

Natürlich sind der Interpretation der deskriptiven Evidenz Grenzen gesetzt. Eine zentrale Einschränkung ist, dass visuelle Evidenz nicht in der Lage ist, den Einfluss von Angebotsbeschränkungen von Nachfrageeinflüssen zu isolieren. Bislang sind wir implizit davon ausgegangen, dass Nachfrageschocks überall gleich ausfallen und, dass Unterschiede in der Zyklizität nur durch Unterschiede in der langfristigen Preiselastizität des lokalen Angebots bedingt sind (und nicht durch Unterschiede

Staaten), entsteht der Eindruck eines ganzheitlichen nationalen Wohnungsmarktes. In der Realität werden die lokalen Märkte jedoch nur vorübergehend von denselben Schocks getrieben. Bracke (2013), der von 1970 bis 201019 OECD-Länder in einem Zeitraum von 40 Jahren erforschte, stellte fest, dass der Median eines vollen Zyklus auf Länderebene etwa 10 Jahre dauerte, sich aber irgendwo zwischen 3 und 20 Jahren bewegte. Der Median eines abgeschlossenen Aufschwunges (Abschwunges) dauert 21 (17) Quartale. Interessanterweise stellte Bracke fest, dass Länder mit längeren Expansionsperioden auch tendenziell längere Kontraktionsperioden aufweisen.

11 Alle vier Metropolregionen sind von Freiflächen umgeben, verfügen über ausreichend bebaubares Land für Wohnzwecke (Hilber und Robert-Nicoud 2013) und sind wenig reguliert (Gyourko et al. 2008 und Saiz 2010). 
im lokalen Konjunkturzyklus auf der Nachfrageseite). In Abb. 1 sind die Nachfrageverschiebungen in beiden Städten A und B identisch. Der einzige Unterschied besteht darin, dass eine Stadt über ein unelastisches langfristiges Angebot verfügt, während in der anderen Stadt ein elastisches Angebot besteht. In der Realität kann die Nachfragevolatilität oder das Ausmaß der lokalen Konjunkturzyklen jedoch je nach Markt erheblich variieren. Wir sind mit dem klassischen ,Identifizierungsproblem“ konfrontiert. Das heißt, wir wissen nicht, ob Preisbewegungen eine Folge von Verschiebungen der Nachfrage- oder der Angebotskurve sind (Hilber 2007). Zudem geben die Abb. 2, 3, 4, 5, 6 und 7 auch keinen Aufschluss darüber, welche Angebotsbeschränkungen (regulatorische, geografische oder topografische) hauptsächlich für die Zyklizität von Immobilienpreisen verantwortlich sind. Lediglich eine rigorose ökonometrische Analyse mit kausaler Identifikation von Effekten kann schlüssig verschiedene angebots- und nachfrageseitige Effekte isolieren.

Im nächsten Abschnitt fasse ich die Ergebnisse der aktuellen empirischen Literatur zusammen, in der unter anderem auch die kausale Rolle von Angebotsbeschränkungen bei der Ermittlung der Immobilienpreise und der Preisdynamik genauer untersucht wurde.

\section{Die Rolle langfristiger Angebotsbeschränkungen für die Immobilienpreiskapitalisierung und die Preisdynamik ${ }^{12}$}

\subsection{Hauspreiskapitalisierung und geografische Angebotsbeschränkungen}

Die Untersuchung der Kapitalisierung fiskalischer Variablen (kommunale Steuern, kommunale öffentliche Dienstleistungen, Subventionen) in Immobilienpreisen geht auf ein wegweisendes Papier von Oates (1969) zurück, der den ersten schlüssigen Nachweis erbrachte, dass steuerliche Unterschiede zu einem großen Teil in unterschiedlichen Immobilienpreisen kapitalisiert werden. Das heißt, dass beispielsweise lokale Grundsteuern unter sonst gleichen Bedingungen lokale Immobilienpreise senken während lokale Ausgaben für öffentliche Schulen diese steigen lassen. ${ }^{13}$

Zahlreiche Studien, die auf Oates (1969) aufbauen, haben seit dem eine bessere Datengrundlage oder verfeinerte Methoden verwendet. ${ }^{14}$ Die meisten Studien, die in der Regel in städtischen oder vorstädtischen Gebieten durchgeführt wurden,

\footnotetext{
12 Dieser Abschnitt baut in wesentlichen Teilen auf Hilber (2017) auf. Für eine eingehendere Diskussion und Überprüfung der relevanten Literatur verweise ich auf dieses Synthese-Papier.

13 Oates (1969) ging davon aus, dass der Nachweis solcher Kapitalisierungseffekte die sogenannte „Tiebout-Hypothese“ stützt. Tiebout (1956) argumentierte, dass die Mobilität der Konsumenten und der zwischenstaatliche Wettbewerb zumindest unter einigen restriktiven Voraussetzungen zu einer effizienten Bereitstellung lokaler öffentlicher Güter führen. Heute herrscht unter den Gelehrten der öffentlichen Finanzen Konsens darüber, dass die Kapitalisierung von fiskalischen Unterschieden in den Immobilienpreisen keinen Test der Tiebout-Hypothese darstellt. Der Nachweis der Kapitalisierung sprich lediglich für die Ansicht, dass die Haushalte für lokale öffentliche Dienstleistungen ,mit den Füßen stimmen“. Für eine eingehendere Diskussion dieses Literaturstrangs siehe Chaudry-Shah (1988), Ross und Yinger (1999) und Hilber (2017).

$14 \mathrm{Zu}$ den Verbesserungen zählen die Verwendung des Boundary Discontinuity Approach (siehe z. B. Basten et al. 2017), die Nutzung von Variationen, die durch natürliche Experimente in Kombination mit einem
} 
bestätigten jedoch die Größenordnung der Ergebnisse von Oates: fiskalische Variablen kapitalisieren sich teilweise bis vollständig in den Immobilienpreisen. Einige Studien fanden sogar Hinweise für eine Überkapitalisierung. Sehr wenige Studien ergaben keine Hinweise auf eine Kapitalisierung: Ein Beispiel ist die Studie von McMillan und Carlson (1977), welchedie Kapitalisierungseffekte lokaler Grundsteuern und lokaler öffentlicher Dienstleistungen in kleinen Städten im ländlichen Wisconsin geschätzt haben. Clark et al. (1993) verwenden Daten für die Region Saskatchewan in Kanada und finden nur sehr schwache Belege für die Hypothese, dass kurzfristige Subventionen in Bodenwerten kapitalisiert werden. Gemeinsam ist diesen beiden Studien, dass Land in beiden Gebieten (Wohngebiet im ländlichen Wisconsin und Ackerland in Saskatchewan) wahrscheinlich sehr elastisch angeboten wird. Dies wurde in den jeweiligen Studien jedoch nicht explizit getestet.

Die These, dass das Ausmaß der Kapitalisierung von fiskalischen Variablen von der langfristigen Preiselastizität des Angebots von Grundstücken oder Wohnraum abhängen könnte, wurde erst in jüngerer Zeit systematisch untersucht. ${ }^{15}$

In einer Reihe neuerer Studien wurde die Rolle geografischer Angebotsbeschränkungen für das Ausmaß der Immobilienpreiskapitalisierung untersucht. Brasington (2002) teilt seine Stichprobe in Häuser innerhalb und am Rande eines Stadtgebiets auf und stellt fest, dass die Kapitalisierung am Rande schwächer ist, wo die Preiselastizität des Immobilienangebots und die Bautätigkeit wahrscheinlich größer sind. Cheshire und Sheppard (2004) stellen fest, dass die Kapitalisierung von Schulqualität in Gebieten mit erhöhter Bautätigkeit niedriger ist. Hilber und Mayer (2009) verwenden einen Instrumentvariablenschätzer in ersten Differenzen sowie Luftbildund Satellitendaten, aus denen für jede Gemeinde in Massachusetts und jeden Schulbezirk in den Vereinigten Staaten der Anteil des bereits bebauten Wohngebiets an der gesamten bebaubaren Fläche berechnet wird. ${ }^{16}$ Ihre Ergebnisse deuten darauf hin, dass geografisch beschränkte Orte (i) eine geringere Preiselastizität des Immobilienangebots und (ii) ein höheres Maß an Immobilienpreiskapitalisierung verschiedener Nachfragefaktoren, einschließlich (instrumentierter) Schulausgaben, aufweisen. Hilber et al. (2011) konzentrieren sich auf die Auswirkungen von regionalen Transfers auf die lokalen Immobilienpreise in England. Ihre Ergebnisse legen nahe, dass Immobilienpreise an Standorten, an denen die Bautätigkeit durch unwegsames Gelände eingeschränkt ist, etwas stärker reagieren.

\subsection{Lokale Regulierung und die Wirkung auf Immobilienmärkte}

Ein anderer Teil der Literatur befasst sich mit regulatorischen Angebotsbeschränkungen und deren Auswirkungen auf die Preiselastizität des Angebotes, das Bau-

\footnotetext{
Difference-in-Differences-Schätzer induziert wurden (siehe z. B. Bogart und Cromwell 2000) oder verfeinerte Instrumental Variable Strategien (siehe z. B. Hilber und Mayer 2009).

15 Hilber (1998, Kapitel 3.3.8) postulierte, dass das Ausmaß der Kapitalisierung von der langfristigen Preiselastizität des Angebots von Grundstücken abhängt. Er hat seine These jedoch nicht empirisch getestet.

16 Land wird als „,nicht bebaubar“ definiert, wenn es als offenes Gewässer, Land mit ewigem Eis, Ödland oder Feuchtgebiet eingestuft wird.
} 
volumen und die Immobilienpreise. ${ }^{17}$ Mayer und Somerville (2000) dokumentieren, dass Metropolregionen mit einer stringenten Regulierung, bis zu $45 \%$ weniger Neubauten und eine um $20 \%$ geringere Preiselastizität des Angebots aufweisen. Green et al. (2005) zeigen, dass die Unterschiede in geschätzten Angebotselastizitäten verschiedener Metropolregionen hauptsächlich auf die unterschiedliche Stärke der Regulierung von Grund- und Bodenmärkten zurückzuführen sind. Insbesondere zeigen die Autoren auf, dass stark regulierte Orte immer eine geringe Preiselastizität des Angebots aufweisen.

Eine Reihe von Studien untersucht die Auswirkungen regulatorischer Eingriffe in den Wohnungsmarkt auf die Immobilienpreise. Malpezzi (1996) liefert in einer frühen Studie Hinweise darauf, dass die Stärke der Regulierung die Immobilienpreise auf statistisch signifikante Weise beeinflusst. Quigley und Raphael (2005) zeigen für kalifornische Städte, dass lokale regulatorische Auflagen positiv mit Immobilienpreisen und Preiswachstumsraten und negativ mit dem Wohnungsneubau korreliert sind.

Glaeser et al. (2005) entwickelten einen anderen Ansatz zur Quantifizierung der Auswirkungen der Regulierung auf den Preis von Wohnraum. Die Grundidee ihres „Regulierungssteuer-“ (regulatory tax) Ansatzes ist einfach. Bei freiem Wettbewerb unter Bauunternehmern und freiem Markteintritt entspricht der Marktpreis für Wohnraum den Durchschnittskosten. Die Grenzkosten steigen mit der Gebäudehöhe. In Abwesenheit von regulatorischen Eingriffen ergibt sich die effiziente Gebäudehöhe als der Punkt, an dem die Grenzkosten eines zusätzlichen Stockwerks dem Marktpreis für Wohnraum entsprechen. Eine Differenz zwischen dem beobachteten Marktpreis und den Grenzkosten kann daher als implizite Steuer der Regulierung interpretiert werden. Glaeser und seine Mitautoren schätzen, dass die Regulierungssteuer in den am stärksten regulierten Metropolregionen der Vereinigten Staaten beträchtlich ist: Durch behördliche Auflagen erhöhen sich die Wohnungspreise demnach um rund $50 \%$ und in Manhattan sogar um deutlich mehr. ${ }^{18}$

Hwang und Quigley (2006) zeigen, dass ähnliche Einkommensschocks Immobilienpreise je nach lokaler Regulierung unterschiedlich beeinflussen. Ein positiver Einkommensschock in Metropolregionen wie Denver mit geringer Regulierung führt nur zu einem moderaten Anstieg der Immobilienpreise. Wäre der Wohnungsmarkt in Denver ähnlich stark reguliert wie derjenige in San Francisco, einer stringent regulierten Stadt, wären die Auswirkungen auf die Immobilienpreise stattdessen erheblich und anhaltend. Folgerichtig ist das zusätzliche Wohnungsangebot, das sich aus einem positiven Einkommensschock ergibt, in Denver größer als in San Francisco.

Saks (2008) verwendet schließlich Informationen aus Umfragen zur Grund- und Bodenmarktregulierung aus den 1970er und 1980er Jahren, um einen Index ab-

\footnotetext{
17 Eine breitere Übersicht über die Messung der Stringenz der Regulierung, die Determinanten der Regulierung und ihre Auswirkungen befindet sich in Gyourko und Molloy (2015).

18 Siehe auch Glaeser und Gyourko (2017) für eine Diskussion der wirtschaftlichen und sozialen Auswirkungen der Regulierungssteuer. Siehe auch die Studie von Cheshire und Hilber (2008), welche die Höhe der Regulierungssteuer für Bürogebäudemärkte in England und andere europäischen Städte quantifiziert haben.
} 
zuleiten, der die Intensität der Regulierung für einzelne Metropolregionen misst. Saks zeigt, dass Standorte mit geringen Auflagen mehr Bauprojekte und geringere Anstiege der Immobilienpreise als Reaktion auf einen positiven Nachfrageschock aufweisen. ${ }^{19}$

\subsection{Determinanten der Preiselastizität des Wohnungsangebots}

Die obige Literatur konzentriert sich entweder auf geografische Angebotsbeschränkungen (Abschn. 3.1) oder auf regulatorische Angebotsbeschränkungen (Abschn. 3.2), um Rückschlüsse auf die Preiselastizität des Wohnraumangebots zu ziehen. Im Gegensatz dazu bezieht Saiz (2010) systematisch die Rolle der Topografie und der Gewässer zusätzlich zu dem regulatorischen Rahmen mit ein, um die Preiselastizität des Wohnungsangebots zu schätzen. Seine Untersuchung umfasst dabei fast 100 Metropolregionen in den Vereinigten Staaten. ${ }^{20}$ Im Ergebnis stellt Saiz fest, dass sowohl geografische als auch regulatorische Beschränkungen die Elastizität des Wohnraumangebots negativ beeinflussen..$^{21}$

Paciorek (2013) verwendet ein dynamisches strukturelles Modell zu Beschreibung von Immobilieninvestitionen, um den Zusammenhang zwischen geografischen und regulatorischen Beschränkungen und der Volatilität der Immobilienpreise zu untersuchen. Angebotsbeschränkungen erhöhen die Preisvolatilität auf zwei Wegen. Erstens verringern strenge regulatorische Auflagen die Elastizität des Angebots, indem sich Baugenehmigungen verzögern und Baukosten erhöhen. Zweitens verringern geografische Beschränkungen durch Gewässer und steile Hänge die durchschnittlichen

${ }^{19}$ Saks (2008) zeigt auch, dass regulatorische Angebotsbeschränkungen die Beschäftigungs- und Lohndynamik beeinflussen. Dies unterstützt die These, dass die Preiselastizität des Wohnungsangebots einen wichtigen Einfluss auf die lokalen Arbeitsmärkte hat, da die Immobilienpreise die Migration beeinflussen.

20 Saiz (2010) berechnet den prozentualen Anteil der Landfläche mit einer Neigung von mehr als $15^{\circ}$ und interpretiert dies als topografische Angebotsbeschränkung mit der Begründung, dass jede Neigung über $15^{\circ}$ schwierig und teuer zu bebauen ist. Er verwendet Satellitendaten, um die Bodenfläche zu bestimmen, die von internen Gewässern und Feuchtgebieten bedeckt ist und verwendet GIS-Techniken, um die von Ozeanen (sowohl den Pazifik als auch den Atlantik) bedeckte Fläche innerhalb eines Radius von $50 \mathrm{~km}$ um den geographischen Mittelpunkt einer Metropolregion zu berechnen. Die beiden letztgenannten MaBe stellen wasserbezogene geografische Angebotsbeschränkungen dar. Zusammengenommen bieten diese drei Beschränkungen einen Proxy für exogen unbebautes Land in einer Metropolregion. Die Intensität der Regulierung wird durch den Wharton Residential Land Use Regulation Index (WRLURI) gemessen - vgl. Gyourko et al. (2008) für Details. Die geschätzten Preiselastizitäten des Angebots wurden von zahlreichen Wissenschaftlern genutzt, um die wirtschaftlichen Auswirkungen des Immobilienangebots in den USA zu untersuchen.

21 Der negative Zusammenhang zwischen geografischen Beschränkungen und der Elastizität des Wohnungsangebots ist intuitiv plausibel. Betrachten wir zunächst einen Extremfall, in dem ein stark nachgefragter Ort vollständig bebaut ist. Dort wird das Angebot an bebaubarem Land nahezu perfekt preisunelastisch sein. Dies liegt daran, dass vorhandene Gebäude, die typischerweise an begehrten Orten gut instandgehalten werden, nur unter hohen Kosten abgerissen und neu errichtet werden können. Es gibt eine Reihe weiterer Erklärungen, weshalb stärker entwickelte Standorte ein weniger preiselastisches Angebot aufweisen. Ein Argument stützt sich auf die Literatur zur Endogenität von Flächennutzungsplänen (,,endogenes Zoning") - siehe die Diskussion weiter unten. Ein zweites Argument leitet sich aus der Realoptionstheorie ab, die davon ausgeht, dass Abriss und Neubebauung kostspielig sind und freies bebaubares Land daher einen Optionswert besitzt. Wenn ein Ort zunehmend entwickelt wird, werden die Opportunitätskosten einer zusätzlichen Einheit - die reale Option - steigen, bei hinreichender Knappheit wahrscheinlich exponentiell. 
Investitionen im Verhältnis zur Größe des vorhandenen Bestands. Pacioreks Untersuchungen bestätigen, dass geografische und regulatorische Beschränkungen dazu führen, dass Immobilien-Investitionen weniger stark auf Nachfrageschocks reagieren, was wiederum die Volatilität der Immobilienpreise verstärkt.

Interessanterweise stößt Saiz (2010) bei der Untersuchung der Zusammenhänge zwischen Geografie, Regulierung und Stadtentwicklung auf einen starken positiven Zusammenhang zwischen lokalen regulatorischen und geografischen Beschränkungen. Das heißt, Metropolregionen mit geografischen Beschränkungen durch Gewässerflächen und Erhebungen weisen typischer Weise auch eine stärkere Regulierung auf. Saiz argumentiert, dass der postive Zusammenhang dadurch entsteht, dass die regulatorischen Beschränkungen endogen auf die Immobilienpreise reagieren (,endogene Zonenplanung"). Sind hohe Immobilienpreise durch geografische Zwänge einmal entstanden, ergeben sich für die Wohnungseigentümer Anreize, sich durch gezieltes Wahlverhalten für eine stärkere regulative Beschränkung des Angebots einzusetzen, um die hohen Vermögenswerte zu schützen (Stichwort ,homevoter hypothesis").

Hilber und Robert-Nicoud (2013) argumentieren, dass diese Interpretation unvollständig ist, da Flächennutzungspläne auch durch gezielte Lobbyarbeit beeinflusst werden können. Daher sollten nebst den Eigennutzern und Mietern (die wählen) auch die Vermieter (Eigentümer, die ihre Immobilien vermieten) und Eigentümer von unbebautem Land (die Lobbying betreiben können) sowie deren Anreize berücksichtigt werden. Eigentümer von bebautem Land - Eigennutzer und Vermieter werden einen Anreiz haben, das Angebot an neuen Wohnimmobilien zu begrenzen, um ihre Vermögenswerte zu schützen und möglicherweise zu vermehren. Eigentümer von unbebautem Land werden im Gegenteil ein Interesse an einem Flächennutzungsplan mit wenigen Vorgaben haben, um die Bebauungskosten niedrig zu halten und die Intensität der Bodennutzung zu maximieren. Ergibt sich nun die Intensität der Regulierung aus einem politischen Kräftemessen zwischen Eigentümern von bebautem und unbebautem Land, würde man erwarten, dass die Eigentümer von bebautem Land sich in stärker bebauten Gebieten eher durchsetzen, da sie dort zahlreicher sind (,influential landowner hypothesis“). ${ }^{22}$ Dies mag auch erklären, warum besonders begehrte Gebiete (wie San Francisco oder New York) strenger reguliert werden: Begehrte Standorte (mit einer stärkeren Nachfrage) sind stärker bebaut und als Ergebnis eines polit-ökonomischen Prozesses stärker reguliert. ${ }^{23}$

\footnotetext{
22 Zahlreiche Studien belegen die „Influential Landowner Hypothesis“. Hilber und Robert-Nicoud (2013) zeigen, dass der Grad der baulichen Entwicklung (lokale Bodenknappheit) in einer Metropolregion in den USA einen kausal positiven Effekt auf die allgemeinen regulatorischen Beschränkungen dieser Metropolregion hat. Rudel (1989) zeigt, dass Gemeinden in Connecticut Gesetze welche die Bodennutzung regulieren umso später verabschiedeten, je weiter sie von New York City entfernt waren und je größer der Anteil an Ackerland war. Strengere Bodennutzungsregulierungen wurden dort implementiert, wo die Landwirtschaft in den 1960er Jahren am stärksten zurückging. In ähnlicher Weise zeigt Fischel (2004), dass Bodennutzungsregulierungen in der Regel zunächst in den Zentren von Großstädten eingeführt werden und dann auf die umliegenden Vororte und Städte ausgeweitet werden. Schließlich schätzen Glaeser et al. (2005) eine sehr hohe „Regulierungssteuer“ (regulatory tax) für Eigentumswohnungen in Manhattan und eine wesentlich geringere regulatorische Belastung für die gesamte Metropolregion.

23 Diese These hat Implikationen für empirische Untersuchungen, welche im Titel des Artikels von Davidoff (2016) pointiert zusammengefasst sind: „Supply Constraints Are Not Valid Instrumental Variables
} 


\subsection{Der Einfluss der Preiselastizität des Angebots auf Grundstückspreise}

Die oben diskutierten Studien untersuchen die Auswirkungen verschiedener Arten von Angebotsbeschränkungen auf die Immobilienpreise. Eine kleine Anzahl von Forschungsarbeiten hat sich stattdessen auf Bodenwerte konzentriert. In Übereinstimmung mit unserer Hypothese 1 dokumentieren Davis und Palumbo (2008), dass die Bodenwerte in Metropolregionen mit einem unelastischeren Angebot volatiler sind. Kuminoff und Pope (2012) stellen fest, dass Randgebiete in Metropolregionen die größte Volatilität bei den Bodenwerten aufweisen. Dies mag daran liegen, dass die Lockerung der Kreditbeschränkungen für Haushalte mit geringerem Einkommen besonders wichtig war, damit sie Häuser am Rand der Vororte kaufen konnten. In ähnlicher Weise haben Kok et al. (2014) die Auswirkungen des lokalen Flächennutzungsplans auf die städtischen Bodenpreise in einer Metropolregion (der San Francisco Bay Area) untersucht, wobei sie für die Effekte von Topografie und Zugang zu Arbeitsplätzen kontrolliert haben. Sie stellen fest, dass Mikrolagen mit strengerer Bodennutzungsregulierung (gemessen an der Anzahl notwendiger Prüfungen zur Erlangung einer Baugenehmigung oder einer Änderung der Flächennutzung) unter sonst gleichen Bedingungen höhere Grundstücks- (und Wohnungs-) preise aufweisen.

\subsection{Angebotsbeschränkungen als Determinante der Reaktion von Immobilienpreisen auf Nachfrageschocks}

Während sich die Autoren der obigen Papiere auf die Wirkung von Angebotsbeschränkungen auf die Preiselastizität des Angebots konzentrieren, untersuchen andere Studien die Wirkung auf die Einkommenselastizität der Immobilienpreise.

Hilber und Vermeulen (2016) nutzen einen Panel-Datensatz englischer Gemeinden (local planning authorities) und verwenden einen Instrumentenvariablenansatz, um die kausalen Auswirkungen von drei verschiedenen Arten lokaler Angebotsbeschränkungen zu identifizieren. Dies sind (i) regulatorische Beschränkungen, die über den Anteil nicht erteilter Baugenehmigungen approximiert werden, (ii) Knappheit von bebaubarem Land, approximiert durch den Anteil bereits bebauter Flächen im Verhältnis zu allen bebaubaren Flächen und (iii) topografische Beschränkungen, approximiert durch die Höhenunterschiede innerhalb einer Gemeinde.

Hilber und Vermeulen zeigen, dass Immobilienpreise in stärker regulierten und geografisch begrenzten Gemeinden viel stärker auf Einkommensschocks und auf lokale Arbeitsnachfrageschocks reagieren. Die Wirkungen regulatorischer Beschränkungen auf die Einkommenselastizität der Immobilienpreise sind in weiten Teilen Englands stark. Dies bestätigt den Eindruck, dass das britische Raumplanungssystem eines der restriktivsten - vielleicht das restriktivste - der Welt ist. ${ }^{24}$ Die Wirkungen

for Home Prices Because They Are Correlated with Many Demand Factors.“ („Angebotsbeschränkungen sind keine gültigen Instrumentvariablen für Immobilienpreise, da sie mit vielen Nachfragefaktoren korrelieren.").

${ }^{24}$ Abb. 8 zeigt, dass das Vereinigte Königreich in den letzten 45 Jahren unter allen OECD-Ländern das stärkste Wachstum der realen Immobilienpreise verzeichnet hat und dies trotz des schleppenden Produktivitätswachstums während eines Großteils dieses Zeitraums. Es folgen an 2. Stelle Neuseeland, an 3. Aus- 


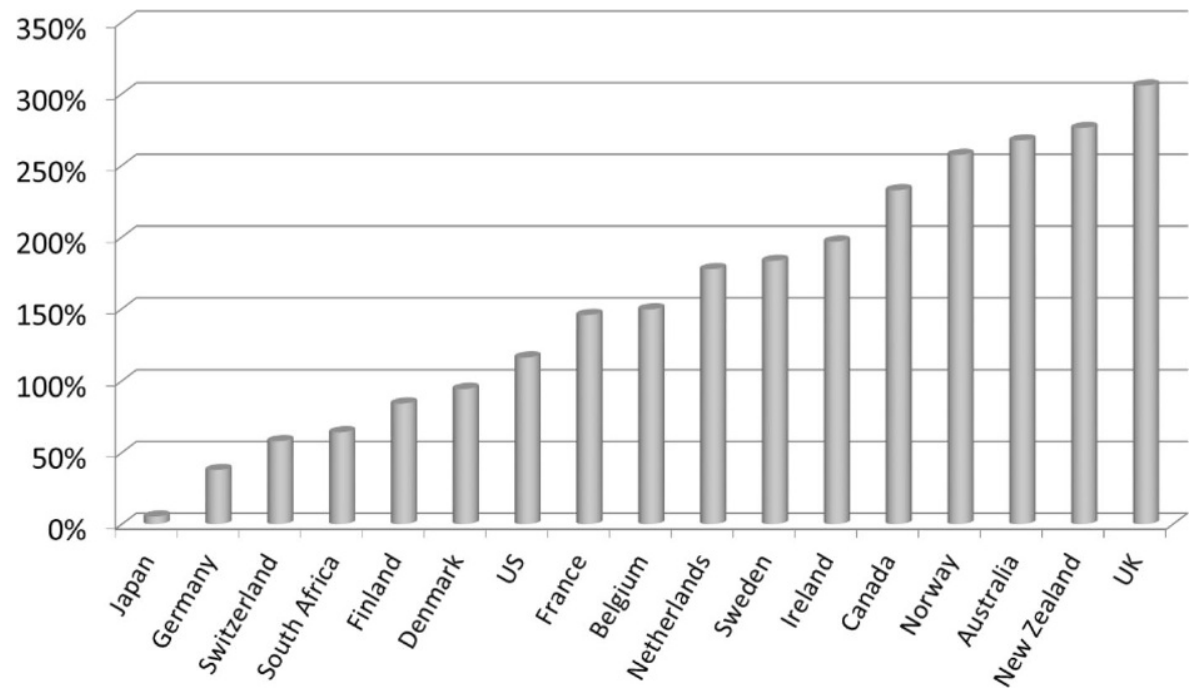

Abb. 8 Reales Wohnimmobilienpreiswachstum in ausgewählten OECD-Ländern von 1970 bis 2015. Quelle: OECD Economic Outlook Database

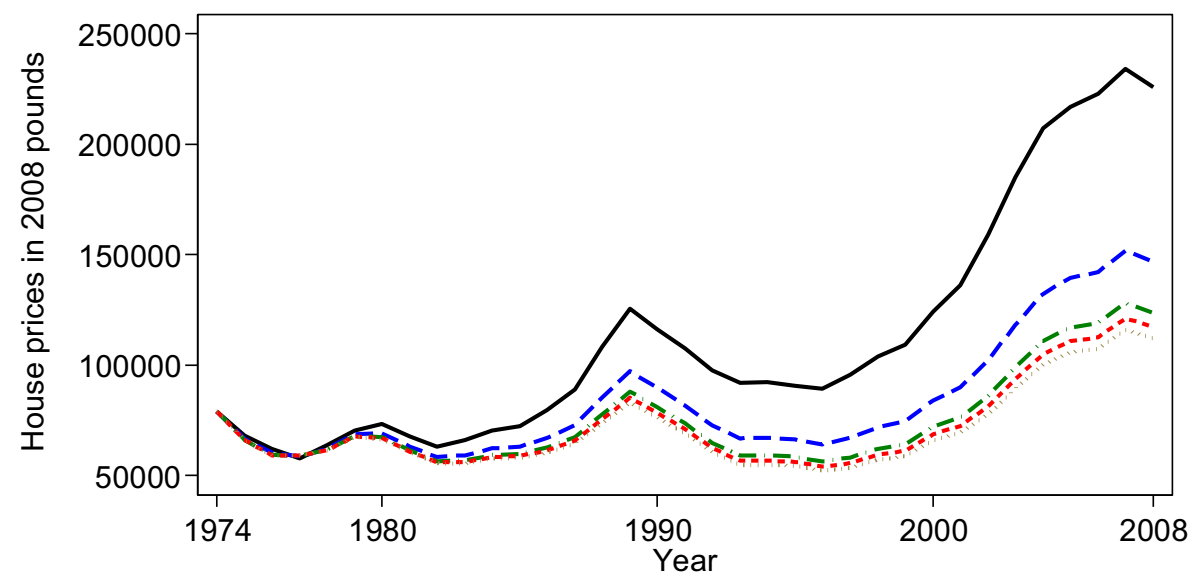

Predicted real house prices in average English LPA

- - - - - Prediction with refusal rate set to zero

- - - - - and share developed set to zero

- and elevation range set to zero

- and independent effect of earnings removed

Abb. 9 Einfluss der Beseitigung von Angebotsbeschränkungen auf die Dynamik der Wohnimmobilienpreise (Quelle: Hilber und Vermeulen 2016) 
der Knappheit an bebaubarem Land beschränkt sich dagegen auf hoch urbanisierte Standorte - hauptsächlich die Greater London Authority. Eine unebene Topografie hat zwar einen statistisch signifikanten Einfluss auf die Einkommenselastizität, in seiner Größenordnung ist der Effekt jedoch eher vernachlässigbar.

Abb. 9 zeigt die relative Bedeutung der verschiedenen Angebotsbeschränkungen auf der Grundlage einer kontrafaktischen Analyse: Wenn Bodennutzungsregulierungen komplett beseitigt würden, wären Immobilienpreise in einer Durchschnittsgemeinde um $35 \%$ tiefer. Immobilienpreise würden um $45 \%$ sinken (um weitere $10 \%$ ), wenn die Knappheit an bebaubarem Land in dieser Durchschnittsgemeinde beseitigt würde. Immobilienpreise wären um $48 \%$ tiefer (weitere 3\%), wenn das Land einer Durchschnittsgemeinde durchgängig eben wäre. Schließlich würden sich die Immobilienpreise halbieren (sich um weitere $2 \%$ verringern), wenn der unabhängige Effekt lokaler Einkommensschwankungen beseitigt würde.

Auch wenn regulatorische Beschränkungen und die Konsequenzen auf Immobilienpreise „hausgemacht“ und im Prinzip reversibel sind, ist es weder praktisch noch wünschenswert, alle derartigen Beschränkungen vollständig zu beseitigen. Im Prinzip dienen regulatorische Eingriffe in den Markt dem wichtigen Ziel, Marktversagen zu korrigieren und können, wenn sie klug angelegt sind, einen Wohlfahrtsgewinn herbeiführen. Zielführender ist daher die Frage, was passieren würde, wenn die restriktivste Region in England (der Südosten) die Regulierungsstringenz der am wenigsten restriktiven, im internationalen Vergleich aber immer noch hochrestriktiven Region (der Nordosten) übernehmen würde? Die Antwort ist, dass die Immobilienpreise um ungefähr $25 \%$ tiefer wären.

Interessanterweise stellen Hilber und Vermeulen (2016) fest, dass die Auswirkungen von Angebotsbeschränkungen während des Aufschwungs größer sind als während der Phasen des Abschwungs. Diese Feststellung deckt sich mit Hypothese 3 und der Ansicht, dass die langfristige Angebotskurve nach unten abknickt (siehe die Diskussion in der Einleitung). In Zeiten stark rückläufiger Nachfrage sollten daher alle Märkte ein ähnlich preisunelastisches Angebot aufweisen, sodass es kaum Unterschiede in der Reaktion der Immobilienpreise auf Einkommens- oder Arbeitsnachfrageschocks geben sollte.

Abb. 9 zeigt ebenfalls, dass die Immobilienpreise auch noch dann eine gewisse Zyklizität aufweisen, nachdem alle Angebotsbeschränkungen und der unabhängige Effekt lokaler Einkommensschwankungen hypothetisch beseitigt sind. Dies legt den Schluss nahe, dass makroökonomische Faktoren eine Rolle spielen, die in der empirischen Spezifikation von Hilber und Vermeulen (2016) über jahresspezifische „fixed effects“ erfasst wurden. So sollten sich die Kreditnachfrage und/oder das Kreditangebot auf nationaler Ebene auf den Hypothekenzins (Preis) und die Verfügbarkeit von Hypothekendarlehen (Menge) auswirken. Die Verfügbarkeit billiger (teurer) Kredite wirkt sich wiederum positiv (negativ) auf die Immobiliennachfrage auf der Makroebene aus, und damit auf die Immobilienpreise im ganzen Land.

tralien und an 5. Stelle Kanada. Alle diese Länder verfügen über viel unbebautes Land für zukünftige Entwicklungen. Keines dieser Länder ist starken geographischen Beschränkungen ausgesetzt, und dennoch ist Wohnraum in den großen Städten Neuseelands, Australiens und Kanadas für viele mittlerweile kaum noch erschwinglich. Gemeinsam ist diesen Ländern, dass es sich um Commonwealth-Länder handelt, deren Raumplanungssystem dem britischen „Entwicklungsskontroll-System“ nachempfunden wurde. 
Favara und Imbs (2015) beleuchten die Auswirkungen einer exogenen Ausweitung von Hypothekendarlehen auf die Immobilienpreise in den USA. Sie nutzen die Deregulierung von US-Niederlassungen zwischen 1994 und 2005 als Instrumentenvariable für das Kreditvolumen und zeigen dabei, dass die Kreditvergabe durch deregulierte Banken zunimmt (nicht jedoch für eine Placebo-Stichprobe). Die nachweislich unterschiedlichen Reaktionen der deregulierten und nicht-deregulierten Banken schließen nachfragebasierte Erklärungen aus (d.h. geringere Anforderungen an Sicherheiten, welche die Kreditnachfrage beeinflussen) und legen nahe, dass das Instrument einen exogenen Schock im Kreditangebot abbildet. Favara und Imbs zeigen, dass dieser Kreditangebotsschock die Nachfrage nach Wohnimmobilien und die Immobilienpreise erhöht. Ihren Schätzungen zufolge erklärt die Deregulierung von Bankfilialen in den USA etwa die Hälfte bis zwei Drittel des beobachteten Anstiegs der Hypothekendarlehen von Geschäftsbanken und ein Drittel bis die Hälfte des Anstiegs der Immobilienpreise. Für diesen Beitrag ist insbesondere relevant, dass ein Kreditangebotsschock die Immobilienpreise in Metropolregionen mit preiselastischem Wohnungsangebot weniger (und den Wohnungsbestand stärker) erhöht. Dies impliziert, dass lokale langfristige Angebotsbeschränkungen nicht nur die Reaktion der lokalen Immobilienpreise auf lokale Nachfrageschocks, sondern auch auf makroökonomische Schocks beeinflussen.

Schließlich haben Oikarinen et al. (2018) in einer neueren Studie die Immobilienpreisdynamik von 70 US-amerikanischen Metropolregionen untersucht. Ihr Hauptaugenmerk liegt auf der Analyse des Zusammenhangs zwischen der Preiselastizität des Wohnungsangebots und der Einkommenselastizität der Preise. Oikarinen und seine Mitautoren stellen fest, dass die langfristige Einkommenselastizität der Immobilienpreise im Durchschnitt 0,81 beträgt, sich jedoch zwischen den Metropolregionen erheblich unterscheidet und in den eher angebotsunelastischen Metropolregionen im Allgemeinen größer ist. Oikarinen et al. dokumentieren auch, dass die Amplitude des Immobilienpreiszyklus und seine Dauer in umgekehrtem Verhältnis zur Preiselastizität des Angebots stehen.

\section{Kurzfristige Angebotsbeschränkungen und endogene Zyklen}

Die bisher diskutierte Evidenz legt nahe, dass räumliche Unterschiede in der Immobilienpreisdynamik gut durch das Zusammenspiel von räumlichen Unterschieden in den langfristigen Angebotsbeschränkungen - sowohl geografischer als auch regulatorischer Natur - und lokalen Wohnungsnachfrageschocks erklärt werden können.

Es gibt jedoch eine wichtige Einschränkung: Nicht alle Märkte, die über ausreichende Flächen bebaubaren Lands und über eine vermeintlich flexible Bodennutzungsregulierung verfügen und somit langfristig ein elastisches Immobilienangebot bieten, weisen auch eine nichtzyklische Preisdynamik auf. Las Vegas und Phoenix sind zwei Beispiele. Die Dynamik der Immobilienpreise dieser beiden Märkte ist in Abb. 10 dargestellt. Die Abbildung zeigt, dass die Immobilienpreise in beiden Märkten bis Anfang 2000 einem ähnlich stabilen Preisverlauf folgten wie in anderen Märkten mit elastischem Immobilienangebot (z. B. Chattanooga, Columbus, Dayton oder Cedar-Rapids). Ab etwa 2002/03 stiegen die Immobilienpreise jedoch plötz- 


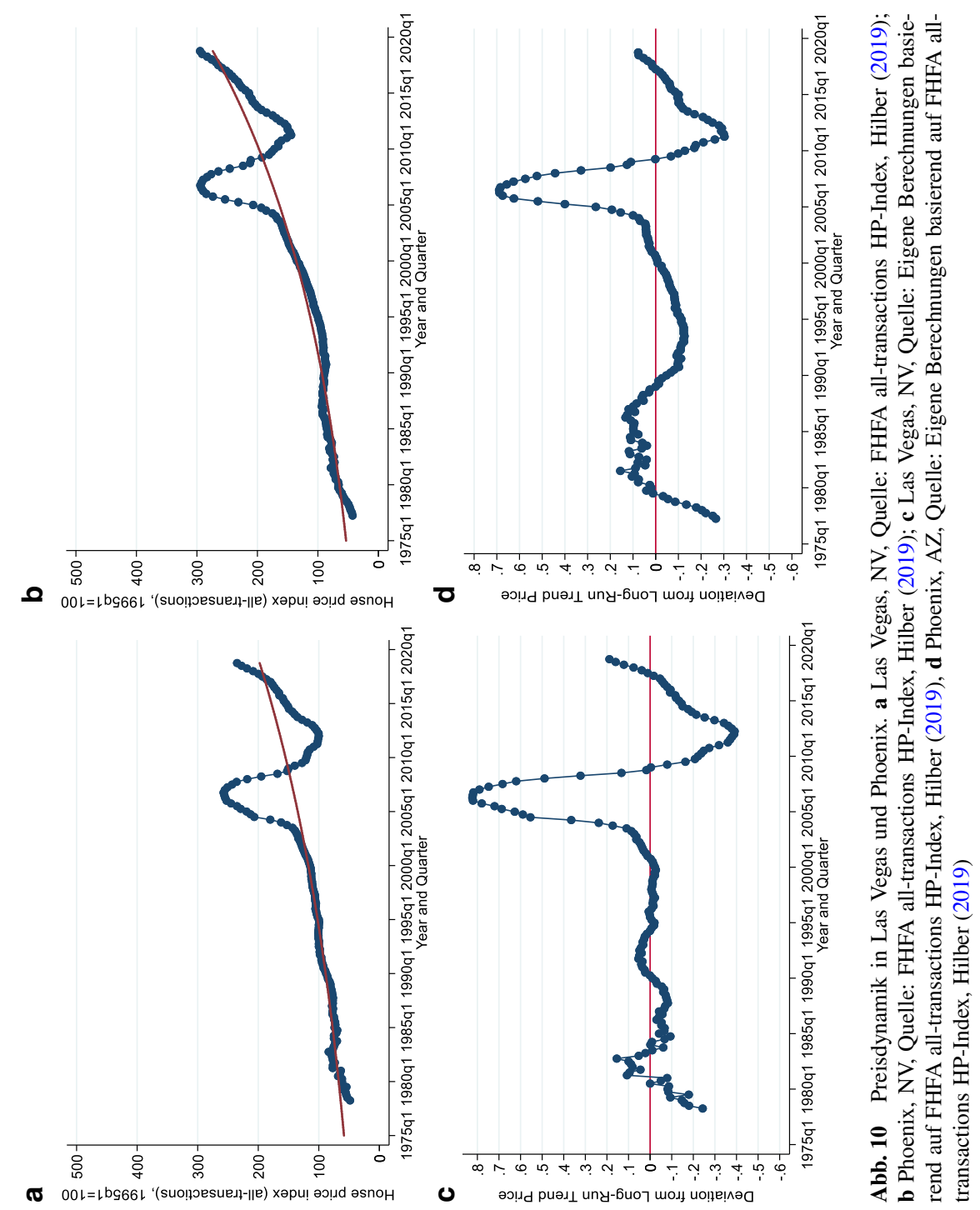




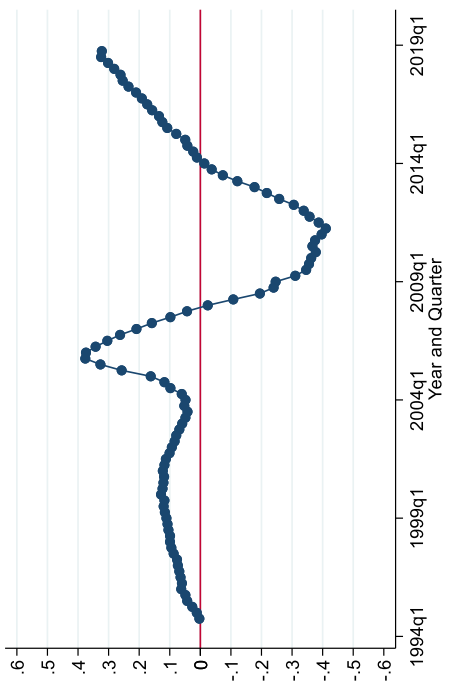

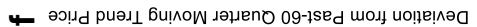

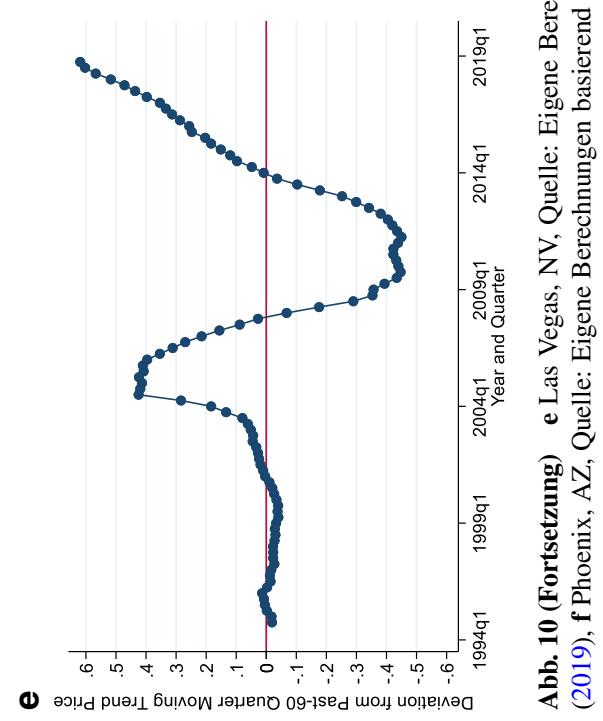


Abb. 11 Kurzsichtige Akteure und verzögerte Reaktion des Angebots

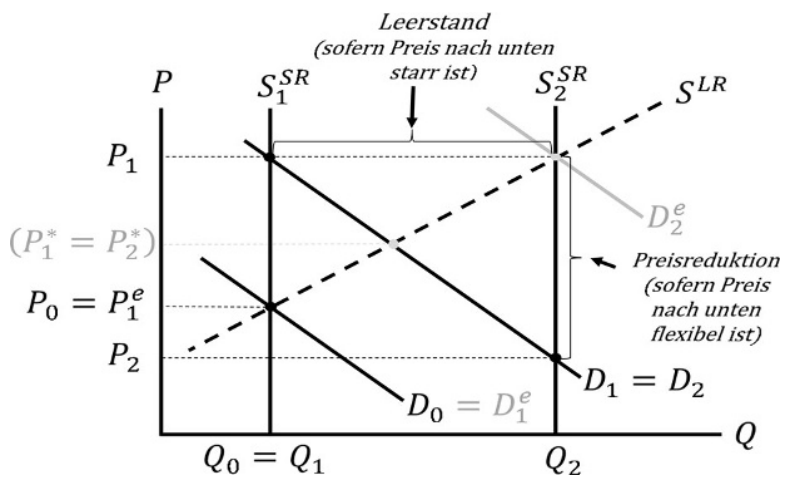

lich sehr schnell an. Zu Spitzenzeiten wichen die Immobilienpreise um über $80 \%$ beziehungsweise um $70 \%$ vom langfristigen Trendpreis ab, was deutlich über den entsprechenden Abweichungen in Los Angeles und San Francisco in den jeweiligen Spitzenzeiten lag. Während die raschen Preiserhöhungen in Las Vegas und Phoenix auffällig waren, waren die Preisstürze, die nach 2006 einsetzten, sogar noch dramatischer. Die Immobilienpreise in Las Vegas gingen von Höchststand zu Tiefststand um rund $70 \%$ zurück und waren nach dem Crash niedriger als vor Beginn des Booms. Darüber hinaus kam es über den Zyklus hinweg zu exzessiver Bauaktivität und hohen Leerstandsquoten. Damit unterscheiden sich die Zyklen stark von Märkten mit unelastischem Angebot (d.h. Boston, Los Angeles, New York oder San Francisco), in denen die Immobilienpreise auf längere Sicht erheblich steigen.

Wie lässt sich diese zunächst überraschende Beobachtung erklären? Eine plausible Erklärung lässt sich daraus ableiten, dass das Wohnungsangebot auf kurze Sicht überall mehr oder weniger preisunelastisch ist. Planung und Bau neuer Einheiten benötigen schließlich Zeit. Anders ausgedrückt, die langfristige Angebotskurve mag elastisch sein, aber die kurzfristige Angebotskurve ist (nahezu perfekt) unelastisch. Diese Situation ist in Abb. 11 dargestellt. Wird eine Metropolregion von einem plötzlichen und unerwarteten positiven Immobiliennachfrageschock getroffen, wird die Bauindustrie nicht sofort alle benötigten Einheiten bereitstellen können, was sich im kurzfristig unelastischen Angebot $\left(S_{1}^{S R}\right)$ zeigt. Die zusätzliche Nachfrage wird sich ausschließlich in höheren Preisen widerspiegeln.

In Abb. 11 wird dieser Prozess auf stilisierte Weise dargestellt, indem eine Wirtschaft betrachtet wird, die normalerweise nicht wächst, d.h. Wirtschaftsakteure im Modell erwarten, dass die Nachfrage nach Wohnraum unverändert bleibt $\left(D_{0}=D_{1}^{e}\right)$. Die Nachfrage verschiebt sich jedoch tatsächlich unerwartet nach $D_{1}$ (ein positiver Nachfrageschock) und die Preise steigen entsprechend von $P_{0}$ auf $P_{1}$ (dies weil die kurzfristige Angebotskurve nahezu perfekt preisunelastisch ist). Nehmen wir nun an, dass Bauunternehmer (und Kreditgeber, die Darlehen bereitstellen) kurzsichtig handeln. Sie beobachten den starken Preisanstieg und beginnen, in der Annahme $P_{1}$ sei der langfristige Gleichgewichtspreis, zu bauen (zu verleihen). Tatsächlich wäre der wohlfahrtsmaximierende langfristige Gleichgewichtspreis $P_{1}^{*}$, da die Nachfrage dann dem langfristigen Angebot entspricht. Die Unternehmer orientieren sich jedoch nicht an diesem Gleichgewichtspreis, sondern an $P_{1}$. Im Ergebnis 
werden Bauunternehmer zu viel bauen. In der Folgeperiode geht das Nachfragewachstum nun auf die ,normale“ Rate zurück (d.h. die Nachfrage ändert sich nicht). Die Bauunternehmer werden feststellen, dass sie tatsächlich zu viel gebaut haben. Sie haben jetzt zwei Möglichkeiten (oder eine Kombination der beiden Möglichkeiten). Entweder sie können die Preise auf $P_{1}$ halten, was zu erheblichem Immobilienleerstand führen wird $\left(\Delta Q=Q_{2}-Q_{1}\right)$, oder sie können den Preis auf den Preis $P_{2}$ senken, um den Markt zu räumen. Anders ausgedrückt, ein einmaliger Schock erzeugt einen ,endogenen Zyklus“; der Preis explodiert zuerst und bricht dann ein. In der Realität (mit steigender Nachfrage im Laufe der Zeit) können Planungs- und Bauzeiten (,construction lags“) in Verbindung mit kurzsichtigen Akteuren auf der Angebotsseite zu oszillierenden Preisen führen, die durch Perioden des Überschießens und Unterschießens gekennzeichnet sind, bis der Preis schließlich wieder auf den langfristigen Gleichgewichtswachstumspfad zurückkehrt.

In Abb. 11 wird eine Situation beschrieben, bei der angebotsseitige Akteure kurzsichtig sind. Endogene Preisschwankungen - ,spekulative Blasen“, die letztendlich zum Einbruch führen - können jedoch auch von kurzsichtigen/enthusiastischen Investoren (Eigennutzern) auf der Nachfrageseite ausgelöst werden. Ausgangspunkt kann erneut ein unerwarteter positiver Nachfrageschock in Verbindung mit einem kurzfristigen unelastischen Angebot sein. Ein alternativer Auslöser könnte ein unerwartetes Preiswachstum auf einem benachbarten Markt (mit unelastischem Angebot ${ }^{25}$ ) in Verbindung mit einem Ansteckungseffekt (oder Spillover-Effekt) sein. Shiller $\left(2000,2014^{26}\right.$ ) argumentiert, dass in einem solchen Kontext Preisblasen (oder irrationale Übertreibungen) auftreten können, wenn sich Anleger massenhaft von einer oberflächlich plausiblen Theorie dazu verleiten lassen, konventionelle Bewertungsmethoden über Bord zu werfen. ${ }^{27}$ Shiller definiert eine Blase als ,eine Situation, in der stetige Berichterstattung über Immobilienpreisanstiege zu zunehmender Begeisterung unter Anlegern führt, welche sich dann in eine Art psychologischer Epidemie [Herdenverhalten] ausbreiten. Dabei werden unter Investoren Geschichten gesponnen, welche die beobachteten Preiserhöhungen rechtfertigen. Nach und nach werden auch skeptische Anleger angesteckt, die eigentlich Zweifel an der Sinnhaftigkeit von Immobilienpreisinvestitionen hegen, aber trotztem investieren, teilweise weil sie andere Investoren um ihre Erfolge beneiden, teilweise weil Sie dem Reiz des Glücksspiels erliegen“ (eigene Übersetzung).

Kurzsichtige Immobilienentwickler und Darlehensgeber in Verbindung mit verzögerter Angebotsreaktion auf Nachfrageschocks und irrationale Übertreibungen von Investoren sind zwei plausible Erklärungsansätze für die außergewöhnliche Dynamik der Immobilienpreise in Las Vegas und Phoenix während der 2000er Jahre. Es handelt sich dabei möglicherweise generell um die zwei intuitiv einleuchtensten Erklärungsansätze für endogene Zyklen. Es ist jedoch wichtig zu betonen, dass es eine Reihe anderer Theorien gibt, die die Preisdynamik von Wohnimmobilien und

\footnotetext{
${ }^{25}$ Es ist zum Beispiel denkbar, dass der Preisboom in Las Vegas ursprünglich durch ein starkes Preiswachstum in einigen benachbarten Metropolregionen mit einer wachsenden Nachfrage und einem unelastischen langfristigen Wohnungsangebot ausgelöst wurde.

26 Shillers Nobelpreisvortrag.

27 Siehe auch Case et al. (2012), die sich speziell mit spekulativem Eigenheimkäuferverhalten befassen.
} 
die Preisdynamik (oder Aspekte davon) in Metropolen wie Las Vegas und Phoenix erklären können.

Nathanson und Zwick (2018) beispielsweise vertreten einen alternativen Ansatz, um zu erklären, warum einige der größten Immobilienpreisbooms in den USA zwischen 2000 und 2006 in Gebieten mit einem elastischen langfristigen Wohnungsangebot auftraten. Sie argumentieren, dass Uneinigkeit über das künftige Nachfragewachstum zu Spekulationen auf dem Grundstücksmarkt führen und die Immobilienzyklen verstärken könnte. ${ }^{28}$ Zyklen können aber zum Beispiel auch durch Suchtheorie und Matching (siehe z. B. Head et al. 2014) oder Optionstheorie in Verbindung mit Investitionsverzögerungen (siehe z. B. Grenadier 1996) erklärt werden. Aspekte der Immobilienpreisdynamik werden auch von Verlustaversion (siehe z. B. Genesove und Mayer 2001) getrieben, und Preisauf- und -abschwünge werden durch das Vorhandensein von Liquiditätsengpässen verstärkt (siehe das theoretische Argument von Ortalo-Magne und Rady 2006 und die Evidenz in Genesove und Mayer 1997). Die detailierte Diskussion dieser Theorien und der unterstützenden Evidenz würde den Rahmen dieses Artikels allerdings sprengen.

\section{Schlussfolgerungen}

Die Dynamik der Wohnimmobilienpreise wird auf komplexe und faszinierende Weise von einer Vielzahl von miteinander zusammenhängenden Faktoren bestimmt. In diesem Artikel habe ich mich auf die Rolle konzentriert, die lokale Angebotsbeschränkungen bei der Erklärung der Immobilienpreisdynamik spielen. Langfristige Angebotsbeschränkungen sind entscheidend, wenn man die Reaktion der Immobilienpreise auf gegebene Nachfrageschocks verstehen möchte. Ökonomische Konjunkturzyklen (mit wiederholten positiven und negativen Nachfrageverschiebungen) führen in Metropolregionen mit starken regulatorischen und geografischen Beschränkungen zu ausgeprägteren Preiszyklen.

Es lassen sich jedoch nicht alle Immobilienpreiszyklen durch nachfrage- und angebotsseitige-Fundamentalfaktoren erklären. De facto haben einige Märkte mit langfristig elastischem Angebot einige der stärksten Auf- und Abschwungsphasen erlebt. Diese Zyklen haben oft wenig mit der Realwirtschaft zu tun. Kurzfristige Angebotsbeschränkungen könnten eine wichtige Rolle bei der Erklärung solcher „endogen getriebenen“ Immobilienzyklen spielen, bei denen ein einmaliger Schock Preisschwankungen mit Über- und Unterschießen auslösen kann, bis die Immobilienpreise schließlich zu ihrem langfristigen Gleichgewichtswachstumspfad zurückkehren. Weitere Forschung ist erforderlich, um die Rolle von kurzfristigen und langfristigen Angebotsbeschränkungen bei der Bestimmung solcher endogen getriebener Zyklen besser zu verstehen.

\footnotetext{
28 Bei Uneinigkeit hinsichtlich künftigen Nachfragewachstums ermöglicht unbebautes Land den Neubau und verstärkt Spekulationen, die zu Auf- und Abschwüngen an Immobilienmärkten führen. Im Modell von Nathanson und Zwick (2018) ziehen mittlere Baubauungsdichten Spekulanten an, die Grundbesitz nur dann halten, wenn sie optimistische Vorstellungen über das künftige Nachfragewachstum haben. Im Marktergebnis sind es dann diese optimistischen Vorstellungen, die die Immobilienpreise bestimmen.
} 
Open Access Dieser Artikel wird unter der Creative Commons Namensnennung 4.0 International Lizenz (http://creativecommons.org/licenses/by/4.0/deed.de) veröffentlicht, welche die Nutzung, Vervielfältigung, Bearbeitung, Verbreitung und Wiedergabe in jeglichem Medium und Format erlaubt, sofern Sie den/die ursprünglichen Autor(en) und die Quelle ordnungsgemäß nennen, einen Link zur Creative Commons Lizenz beifügen und angeben, ob Änderungen vorgenommen wurden.

\section{Literatur}

Arnott R, Stiglitz J (1979) Aggregate land rents, expenditure on public goods, and optimal city size. Q J Econ 93:471-500

Basten C, v. Ehrlich M, Lassmann A (2017) Income taxes, sorting and the costs of housing: evidence from municipal boundaries in Switzerland. Econ J 127:653-687

Bogart WT, Cromwell BA (2000) How much is a neighborhood school worth? J Urban Econ 47:280-305

Bracke P (2013) How long do housing cycles last? A duration analysis for 19 OECD countries. J Hous Econ 22:213-230

Brasington DM (2002) Edge versus center: finding common ground in the capitalization debate. J Urban Econ 52(3):524-541

Case K, Shiller R, Thompson AK (2012) What have they been thinking? Homebuyer behavior in hot and cold markets. Brookings Pap Econ Act 43(2):265-315

Chaudry-Shah A (1988) Capitalization and the theory of local public finance: an interpretive essay. J Econ Surv 2:209-243

Cheshire P, Hilber CAL (2008) Office space supply restrictions in Britain: the political economy of market revenge. Econ J 118(529):F185-F221

Cheshire P, Sheppard S (2004) Capitalising the value of free schools: the impact of supply characteristics and uncertainty. Econ J 114:F397-F424

Clark SJ, Klein KK, Thompson SJ (1993) Are Subsidies Capitalized into Land Values? Some Time Series Evidence from Saskatchewan. Canadian J of Agricultural Economics 41:155-168

Davidoff T (2016) Supply constraints are not valid instrumental variables for home prices because they are correlated with many demand factors. Crit Finance Rev 5:177-206

Davis MA, Palumbo MG (2008) The price of residential land in large U.S. cities. J Urban Econ 63(1):352-384

Favara G, Imbs J (2015) Credit supply and the price of housing. Am Econ Rev 105(3):958-992

Fischel WA (2004) An economic history of zoning and a cure for its exclusionary effects. Urban Stud 41(2):317-340

Genesove D, Mayer C (1997) Equity and time to sale in the real estate market. Am Econ Rev 87:255-269

Genesove D, Mayer C (2001) Loss aversion and seller behavior: evidence from the housing market. Q J Econ 116:1233-1260

Glaeser E, Gyourko J (2017) The economic implications of housing supply. NBER working paper, Bd. 23833 (September)

Glaeser EL, Gyourko J (2005) Urban decline and durable housing. J Polit Econ 113(2):345-375

Glaeser EL, Nathanson CG (2017) An extrapolative model of house price dynamics. J Financial Econ 126:147-170

Glaeser EL, Gyourko J, Saks RE (2005) Why is Manhattan so expensive? Regulation and the rise in housing prices. J Law Econ 48(2):331-369

Green RK, Malpezzi S, Mayo SK (2005) Metropolitan-specific estimates of the price elasticity of supply of housing, and their sources. Am Econ Rev Pap Proc 95(2):334-339

Grenadier S (1996) The strategic exercise of options: development cascades and overbuilding in real estate markets. J Finance 51:1653-1679

Gyourko J, Molloy R (2015) Regulation and housing supply. In: Duranton G, Henderson V, Strange W (Hrsg) Handbook of regional and urban economics, Bd. 5. Elsevier, Amsterdam, S 1289-1337

Gyourko J, Saiz A, Summers A (2008) A new measure of the local regulatory environment for housing markets: the Wharton residential land use regulatory index. Urban Stud 45(3):693-729

Head A, Lloyd-Ellis H, Sun H (2014) Search, liquidity, and the dynamics of house prices and construction. Am Econ Rev 104(4):1172-1210

Hilber CAL (1998) Auswirkungen staatlicher Massnahmen auf die Bodenpreise: eine theoretische und empirische Analyse der Kapitalisierung. Rüegger, Zürich

Hilber CAL (2007) Der Einfluss von Preisänderungen auf Angebot und Nachfrage von Immobilien: Theorie, empirische Evidenz und Implikationen. Z Immobilienökonomie 1(2007):5-20 
Hilber CAL (2017) The economic implications of house price capitalization: a synthesis. Real Estate Econ 45(2):301-339

Hilber CAL, Mayer CJ (2009) Why do households without children support local public schools? Linking house price capitalization to school spending. J Urban Econ 65(1):74-90

Hilber CAL, Robert-Nicoud F (2013) On the origins of land use regulations: theory and evidence from US metro areas. J Urban Econ 75(1):29-43

Hilber CAL, Vermeulen W (2016) The impact of supply constraints on house prices in England. Econ J 126(591):358-405

Hilber CAL, Lyytikäinen T, Vermeulen W (2011) Capitalization of central government grants into local house prices: panel data evidence from England. Reg Sci Urban Econ 41:394-406

Hilber, CAL (2019) Lectures on real estate cycles, LSE Course GY457 Applied Urban and Regional Economics, LSE mimeo

Hwang M, Quigley J (2006) Economic fundamentals in local housing markets: evidence from U.S. metropolitan regions. J Reg Sci 46(3):425-453

Kok N, Monkkonen P, Quigley JM (2014) Land use regulations and the value of land and housing: an intrametropolitan analysis. J Urban Econ 81:136-148

Kuminoff NV, Pope JC (2012) The value of residential land and improvements during the great housing boom and bust. Land Econ 89(1):1-29

Malpezzi S (1996) Housing prices, externalities, and regulation in U.S. metropolitan areas. J Hous Res 7:209-241

Mayer CJ, Somerville CT (2000) Land use regulation and new construction. Reg Sci Urban Econ 30:639-662

McMillan ML, Carlson R (1977) The effects of property taxes and local public services upon residential property values in small Wisconsin cities. Am J Agric Econ 59(1):81-87

Nathanson CG, Zwick E (2018) Arrested development: theory and evidence of supply-side speculation in the housing market. J Finance 73(6):2587-2633

Oates WE (1969) The effects of property taxes and local public spending on property values: an empirical study of tax capitalization and the Tiebout hypothesis. J Polit Econ 77(6):957-971

Oikarinen E, Bourassa SC, Hoesli M, Engblom J (2018) U.S. metropolitan house price dynamics. J Urban Econ 105:54-69

Ortalo-Magne F, Rady S (2006) Housing market dynamics: on the contribution of income shocks and credit constraints. Rev Econ Stud 73:459-485

Paciorek A (2013) Supply constraints and housing market dynamics. J Urban Econ 77:11-26

Quigley JM, Raphael S (2005) Regulation and the high cost of housing in California. Am Econ Rev Pap Proc 95(2):323-328

Ross SL, Yinger J (1999) Sorting and voting: a review of the literature on urban public finance. In: Cheshire P, Mills ES (Hrsg) The handbook of urban and regional economics, Bd. 3. North-Holland, Amsterdam

Rudel TK (1989) Situations and strategies in American land-use planning. Cambridge University Press, Cambridge

Saiz A (2010) The geographic determinants of housing supply. Q J Econ 125(3):1253-1296

Saks R (2008) Job creation and housing construction: constraints on metropolitan area employment growth. J Urban Econ 64:178-195

Shiller RJ (2000) Irrational exuberance, 1. Aufl. Princeton University Press, Princeton

Shiller RJ (2014) Speculative asset prices. Am Econ Rev 104(6):1486-1517

Sinai T, Souleles NS (2005) Owner-occupied housing as a hedge against rent risk. Q J Econ 120(2):763-789

Sinai T, Souleles NS (2013) Can owning a home hedge the risk of moving? Am Econ J Econ Policy 5(2):282-312

Tiebout CM (1956) A pure theory of local expenditures. J Polit Econ 64(5):416-424 